\title{
The Source Equivalence Acceleration Method
}

\author{
Matthew S. Everson and Benoit Forget \\ Massachusetts Institute of Technology \\ 77 Massachusetts Ave., Cambridge, MA 02139 \\ meverson@mit.edu; bforget@mit.edu
}

\begin{abstract}
Fine-group whole-core reactor analysis remains one of the long sought goals of the reactor physics community. Such a detailed analysis is typically too computationally expensive to be realized on anything except the largest of supercomputers. Recondensation using the Discrete Generalized Multigroup (DGM) method, though, offers a relatively cheap alternative to solving the fine group transport problem. DGM, however, suffered from inconsistencies when applied to high-order spatial methods. While an exact spatial recondensation method was developed and provided full spatial consistency with the fine group problem, this approach substantially increased memory requirements for realistic problems. The method described in this paper, called the Source Equivalence Acceleration Method (SEAM), forms a coarse-group problem which preserves the fine-group problem even when using higher order spatial methods. SEAM allows recondensation to converge to the fine-group solution with minimal memory requirements and little additional overhead. This method also provides for consistency when using different spatial methods and angular quadratures between the coarse group and fine group problems. SEAM was implemented in OpenMOC, a 2D MOC code developed at MIT, and its performance tested against Coarse Mesh Finite Difference (CMFD) acceleration on the C5G7 benchmark problem and on a 361 group version of the problem. For extremely expensive transport calculations, SEAM was able to outperform CMFD, resulting in speed-ups of 20-45 relative to the normal power iteration calculation.
\end{abstract}

Keywords: Nonlinear Acceleration; Coarse Mesh Finite Difference; Discrete Generalized Multigroup; Subgroup Decomposition; Cross Section Recondensation; Method of Characteristics

\section{Introduction}

Achieving high fidelity neutronics analysis is of utmost importance in analyzing the performance and safety of reactor designs. Since quantities such as power density are dependent on the neutron distribution, accurate predictions of the neutron flux in a reactor are key to performing 
steady-state and transient safety analyses. These analyses are conducted by solving the neutron transport equation.

Ideally, this equation would be solved exactly for a given 3D reactor model including a detailed representation of all reactor internals and their corresponding cross section data. Such solutions are very expensive computationally especially when one tries to reduce the approximations introduced in the energy condensation process which can require up to 1000's of energy groups. It is thus imperative to make proper use of low-order acceleration models to reduce the computational cost and provide a platform for fluids and fuel performance coupling. The current state-of-the-art in acceleration usually involves some form of non-linear acceleration [1][2][3][4][5] which focuses on spatial acceleration for high dominance ratio cores or the generalized multigroup method with recondensation, a non-linear approach that aims at high dimension energy problems. [6][7][8][9] One of the main problems with generalized multigroup and recondensation is that the nonlinear iteration is not consistent with the fine group problem when using high order spatial methods, meaning the nonlinear iteration does not converge to the fine group solution. [10][11] Previous work modifying the generalized multigroup equations produced full consistency with the fine group problem but came with a substantial increase in memory requirements. To overcome this limitation, this paper proposes a new nonlinear acceleration method as the primary means of producing high fidelity solutions in an efficient manner for high energy and angular dimensionality problems.

\section{Review of Nonlinear Acceleration}

Nonlinear acceleration methods rely on an iterative approach in which full transport sweeps are used to create an equivalent yet cheaper version of the problem. These cheaper versions can be formed using spatial homogenization, energy condensation and/or a low order angular approximation. The solutions from these equivalent problems are then used to reduce the total number of full transport sweeps required to converge the fission source. This provides a relatively cheap process by which the true solution of the full problem can be obtained.

\subsection{Coarse Mesh Finite Difference Diffusion and Coarse Mesh Rebalance}

Coarse mesh finite-difference (CMFD) diffusion is a widely used nonlinear acceleration scheme in reactor physics. [1][3][5] In this approach, the high order angular problem is represented by a low-order diffusion problem. Equivalence is enforced through calculation of nonlinear diffusion coefficient correction factors (diffusion correction factors for short). Equivalence can be maintained across spatial homogenization and energy condensation. The diffusion correction factors are calculated according to Equation (2.1) when incorporating energy condensation simultaneously with spatial homogenization.

$$
\widetilde{D}_{c+1 / 2, g}=-\frac{J_{c+1 / 2, g}+\widehat{D}_{c+1 / 2, g}\left(\phi_{c+1, g}-\phi_{c, g}\right)}{\phi_{c+1, g}+\phi_{c, g}}
$$


Definitions for each quantity are provided below:

- $\phi_{c, g}$ is the scalar flux for coarse mesh cell $c$ and coarse group $g$.

- $\widehat{D}_{c+1 / 2, g}$ is the effective diffusion coefficient between coarse mesh cells $c$ and $c+1$ for coarse group $g$.

- $J_{c+1 / 2, g}$ is the net surface current across the surface spanning coarse mesh cells $c$ and $c+1$ for coarse group $g$.

The procedure highlighted in Equation (2.1) takes into account energy condensation to create a cheaper version of a fine group transport calculation through the simultaneous spatial homogenization and multigroup collapse of scattering, fission, total cross sections, diffusion coefficients and scalar fluxes. [12] An example of this procedure is provided for the fission cross section in Equation (2.2).

$$
v \Sigma_{F, c, g}=\sum_{K \in g} \sum_{f \in c} v \Sigma_{F, f, K} \phi_{f, K} d_{f} / \sum_{K \in g} \sum_{f \in c} \phi_{f, K} d_{f}
$$

The summations take place across the individual fine groups within a coarse group $g$ and are indexed using $K$. $f$ is used to denote each of the fine mesh cells homogenized into coarse mesh cell $c . d_{f}$ is the length of fine mesh cell $f$ in a $1 \mathrm{D}$ problem.

Once the diffusion correction factors are calculated for each surface, a set of finite-difference diffusion equations are solved to provide the new fluxes for each of the coarse meshes. The converged coarse mesh fluxes are then used to update the fine mesh fine group fluxes, thereby accelerating the transport problem.

An alternative to CMFD is the Coarse Mesh Rebalance (CMR) method. [2][4] In this case, neutron conservation is maintained directly through the neutron balance equation by multiplying the surface currents at coarse mesh cell boundaries by a set of rebalance factors. [13] Application to a 1D problem is shown in Equation (2.3) with energy condensation included.

$$
\left(J_{c, g}^{+}+J_{c, g}^{-}+\sum_{f \in c} d_{f} \Sigma_{T, f, g} \phi_{f, g}\right) R_{c, g}-J_{c-1, g}^{+} R_{c-1, g}-J_{c+1, g}^{-} R_{c+1, g}=\sum_{f \in c} d_{f} Q_{c, g}
$$

Definitions for each quantity are provided below:

- $J_{c, g}^{+/-}$is partial surface current for coarse group $g$ exiting coarse mesh cell $c$ from the right and left surfaces respectively.

- $R_{c, g}$ is the rebalance factor for coarse mesh cell $c$ and coarse group $g$.

- $Q_{c, g}$ is the source for coarse mesh cell $c$ and coarse group $g$.

Equation (2.3) forms a series of equations from which the rebalance factors can be solved. The rebalance factors are then used to update the fine mesh fine group cell-averaged scalar fluxes and accelerate the transport problem.

However, it is important to note that both CMFD and CMR suffer from conditional stability issues. Both methods suffer from instabilities for optically thick meshes, but only CMFD 
appears to be stable for optically thin coarse meshes. [1] If CMFD is able to converge for an optically thick problem, little to no acceleration is observed. [12] In many applications of CMFD, a dampening factor is applied to prevent instabilities from growing and causing divergence. The dampening factor prevents overshoot of the accelerated scalar fluxes by limiting how quickly the nonlinear diffusion coefficient correction factor from Equation (2.1) is updated. This damping factor is largely problem dependent but a reasonable value can be applied across a number of different problems. [14] Partial current Coarse Mesh Finite Difference (pCMFD) is a more recent development which has also been shown to improve stability. This approach adds a second degree of freedom to CMFD by calculating two partial current corrective factors at the boundaries of a coarse mesh instead of forming a single net current corrective factor. [1][13]

While multigroup collapse can be and has been incorporated into CMFD and CMR, the primary focus of acceleration for both methods is typically placed on the space-angle problem.

\subsection{Exact Recondensation}

DGM differs from these methods in that its primary focus is on accelerating the spectral problem. This approach is essentially a discrete form of the Generalized Energy Condensation (GEC) method, which was the first method to apply a transformation to the original fine group equations using a series of continuous polynomial functions in energy. [7] On the other hand, DGM uses discrete basis functions to represent the fine group angular flux as angular flux moments according to Equation (2.4). [8] In this equation, $\psi_{K}(\vec{r}, \vec{\Omega})$ is the angular flux for fine group $K$ in coarse group $g, P_{i, K}$ is the $i^{\text {th }}$ discrete basis function evaluated at fine group $K$ and $\psi_{i, g}(\vec{r}, \vec{\Omega})$ is the $i^{\text {th }}$ angular flux moment in coarse group $g$.

$$
\psi_{i, g}(\vec{r}, \vec{\Omega})=\sum_{K \in g} P_{i, K} \psi_{K}(\vec{r}, \vec{\Omega})
$$

The use of discrete basis functions to represent the fine group fluxes better matches the discrete nature of the cross section data. The fine group angular flux can be expanded in terms of these angular flux moments within the multigroup transport equation using Equation (2.5). $a_{i}$ is the normalization constant for the $i^{\text {th }}$ discrete basis function and $N_{g}$ is the total number of fine groups in coarse group $g$, which is equivalent to the total number of moments in coarse group $g$.

$$
\psi_{K}(\vec{r}, \vec{\Omega})=\sum_{i=0}^{N_{g}-1} a_{i} P_{i, K} \psi_{i, g}(\vec{r}, \vec{\Omega})
$$

After this expansion, the resulting equations are coupled to all the moments. To decouple these flux moments, the equations are multiplied by the $i^{\text {th }}$ discrete basis function and summed. This process produces the DGM moment equations shown in Equation (2.6). Once solved, these DGM equations provide a set of flux moments which can then be used to reconstruct fine group fluxes. [8] 


$$
\begin{aligned}
\vec{\Omega} \cdot \nabla \psi_{i, g}(\vec{r}, \vec{\Omega}) & +\Sigma_{T, g}(\vec{r}, \vec{\Omega}) \psi_{i, g}(\vec{r}, \vec{\Omega})+\delta_{i, g}(\vec{r}, \vec{\Omega}) \psi_{0, g}(\vec{r}, \vec{\Omega}) \\
& =\frac{1}{4 \pi} \sum_{g^{\prime}=1}^{G} \Sigma_{s, g^{\prime} \rightarrow i, g}(\vec{r}) \phi_{g^{\prime}}(\vec{r})+\frac{\chi_{i, g}(\vec{r})}{4 \pi k_{e f f}} \sum_{g^{\prime}=1}^{G} v \Sigma_{f, g^{\prime}}(\vec{r}) \phi_{g^{\prime}}(\vec{r})
\end{aligned}
$$

One important note is that the fine group cross sections have also been replaced with moment cross sections. Since the fine group cross sections were expanded in conjunction with the fine group scalar fluxes in order for all quantities to be represented using the same basis functions. The resulting moment cross sections bare striking similarity to the normal multigroup collapse scheme except that discrete basis functions are applied to the outgoing fine groups. The moment scattering cross section is provided as an example in Equation (2.7). This quantity can be thought of as the likelihood of neutrons scattering from coarse group $g$ ' to the $i^{\text {th }}$ moment of coarse group $g$.

$$
\Sigma_{s, g^{\prime} \rightarrow i, g}=\sum_{K \in g} \sum_{L \in g^{\prime}} P_{i, K} \Sigma_{s, L \rightarrow K} \phi_{L}^{r} / \sum_{L \in g^{\prime}} \phi_{L}^{r}
$$

An additional term is added into Equation (2.6) to account for the fact that the coarse group total cross section, $\Sigma_{T, g}(\vec{r}, \vec{\Omega})$, is used across all $i$ moment equations within coarse group $g$. To maintain equivalence with the original fine group problem, the $\delta$ term is used to account for changes in the total reaction rate when the coarse group total cross section is used in place of the moment total cross section. The $\delta$ term incorporates a correction to the left-hand side of the DGM equations by collapsing the difference between the fine group and coarse group total cross sections according to Equation (2.8). This term is then moved to the RHS of the transport equation and treated as an angularly dependent source term.

$$
\delta_{i, g}(\vec{r}, \vec{\Omega})=\sum_{K \epsilon g} P_{i, K} \psi_{K}(\vec{r}, \vec{\Omega})\left(\Sigma_{T, K}(\vec{r})-\Sigma_{T, g}(\vec{r}, \vec{\Omega})\right) / \sum_{K \in g} \psi_{K}(\vec{r}, \vec{\Omega})
$$

Once Equation (2.6) is solved, the fine group flux can be reconstructed using the flux moments. Successive reconstruction of the fine group flux and recollapsing of the coarse group cross sections forms a nonlinear acceleration method called recondensation. Unfortunately, this nonlinear approach is conditionally stable when using fixed point iteration. [15] Stability can be obtained through under-relaxation by a procedure called the Krasnoselskij iteration. [16] However, no particular spatial method (e.g. step difference, diamond difference, step characteristics) was applied in the original derivation of DGM. Initially, DGM was derived from the multigroup transport equation before a spatial method was applied. The result was a $\delta$ term neglecting the assumptions of the spatial method applied after deriving DGM. While the original $\delta$ term was generalized in this case and was applicable to any spatial method, full consistency during recondensation was only observed for methods assuming a flat angular flux across each cell. For methods like step characteristics, which assume an exponential shape to the angular flux, DGM was not equivalent to the original fine group equations and recondensation did not converge to the fine group solution. Therefore, a $\delta$ term was formed specifically for 1-D step 
characteristics by deriving a set of DGM equations from the spatially discretized form of the multigroup transport equation. [11] Equation (2.9) shows the DGM equations across a single cell for step characteristics in 1D.

$$
\begin{gathered}
\psi_{\text {out }, l, i, g}=A_{l, g} \psi_{i n, l, i, g}+B_{l, g} Q_{i, g}+\delta_{l, i, g}^{S C, i o} \overline{\psi_{l, g}} \\
\overline{\psi_{l, l, g}}=\frac{\mu_{l}}{d}\left(B_{l, g} \psi_{i n, l, i, g}+C_{l, g} Q_{i, g}\right)+\delta_{l, i, g}^{S C, a v g} \overline{\psi_{l, g}}
\end{gathered}
$$

Definitions for each quantity are provided below:

- $\quad \psi_{i n, l, i, g}$ is the incoming angular flux for polar angle $l$ and moment $i$ in coarse group $g$.

- $\psi_{o u t, l, i, g}$ is the outgoing angular flux for polar angle $l$ and moment $i$ in coarse group $g$.

- $\overline{\psi_{l, l, g}}$ is the cell-averaged angular flux for polar angle $l$ and moment $i$ in coarse group $g$.

- $Q_{i, g}$ is the source for moment $i$ in coarse group $g$.

$A_{l, g}, B_{l, g}$, and $C_{l, g}$, are referred to as transport coefficients and are defined using the coarse group total cross section. [10] The definitions of the transport coefficients are dependent on the spatial method used to solve the transport equation. In the case of 1D step characteristics, the coarse group transport coefficients are defined according to Equation (2.10).

$$
\begin{gathered}
A_{l, g}=e^{-\Sigma_{T, l, g} d} / \mu_{l} \\
B_{l, g}=\frac{1-A_{l, g}}{\Sigma_{T, l, g}} \\
C_{l, g}=\frac{\frac{d}{\mu_{l}}-B_{l, g}}{\Sigma_{T, l, g}}
\end{gathered}
$$

Although the exact $\delta$ terms defined in Equation (2.11) for step characteristics allowed recondensation to converge to the fine group solution, this achievement came at the cost of large memory requirements.

$$
\begin{aligned}
& \delta_{l, i, g}^{S C, i o}=\frac{\overline{\overline{\delta A_{l, g, l \jmath}}} \overline{\psi_{l n, l, j, g}}+\overline{\overline{\delta B_{l, g, l \jmath}}} \overline{Q_{J, g}}}{\overline{\psi_{l, g}}} \\
& \delta_{l, i, g}^{S C, a v g}=\frac{\frac{\mu_{l}}{d}\left(\overline{\overline{\delta B_{l, g, l]}}} \overline{\psi_{l n, l, j, g}}+\overline{\overline{\delta C_{l, g, l]}}} \overline{Q_{J, g}}\right)}{\overline{\psi_{l, g}}}
\end{aligned}
$$

For the exact $\delta$ term to be correctly defined, the incoming angular flux at every cell face must be stored in order to calculate this quantity on the fly. Since these quantities must be store for each angle, cell face and moment, storage can quickly get out of hand for a realistic problem.

Therefore, this method was considered impractical for achieving the stated goal of full core analysis with fine energy resolution. 


\subsection{Subgroup Decomposition}

The Subgroup Decomposition Method (SGD) developed at Georgia Tech is, at its heart, a simplification of the DGM formulation. [6] Instead of applying a transformation using discrete basis functions, this method conducts the normal coarse group collapse and a second collapse on only the incoming energy group side of the scattering and fission cross sections. An initial guess on the fine group flux is used to produce a set of coarse group cross sections and a set of what are called SGD cross sections.

Whereas DGM forms moment scattering cross sections according to Equation (2.7), SGD produces different scattering cross sections which are only collapsed with respect to the incoming neutron energy group. This can be seen in the SGD scattering cross sections defined in Equation (2.12).

$$
\Sigma_{s, g^{\prime} \rightarrow K}=\sum_{L \in g^{\prime}} \Sigma_{S, L \rightarrow K} \phi_{L} / \sum_{L \in g^{\prime}} \phi_{L}
$$

With these SGD scattering cross sections, a fine group scatter source can be produced using only a coarse group scalar flux. The accuracy of the scatter source produced with the coarse group flux, though, is dependent on the accuracy of the fine group flux used to collapse the fine group scattering cross sections.

The SGD fission cross sections are equivalent to coarse group fission cross sections. However, the fission spectrum is left uncollapsed. Both of these cross sections are referred to as "subgroup decomposition cross sections". These SGD cross sections allow one to build a fine group source using a coarse group flux. Equation (2.13) provides an example for an isotropic fine group source in $1 \mathrm{D}$.

$$
Q_{K}=\frac{1}{2} \sum_{g^{\prime}} \Sigma_{s, g^{\prime} \rightarrow K} \phi_{g^{\prime}}+\frac{\chi_{K}}{2 k_{e f f}} \sum_{g^{\prime}} v \Sigma_{f, g^{\prime}} \phi_{g^{\prime}}
$$

The next step is to conduct a fine group sweep on the fine group source built using the coarse group scalar fluxes. This transport sweep is referred to as the "decomposition sweep". [6] This is slightly different from DGM in that DGM solves a transformed version of the fine group problem whereas SGD directly solves the fine group equations. After the decomposition sweep, a new fine group flux is obtained and is used to recondense both the coarse group cross sections and subgroup decomposition cross sections. This procedure then drives the initial fine group flux guess towards the fine group solution with minimal computational effort.

SGD has shown promise in terms of providing computationally cheap improvements to a coarse group solution with low memory requirements relative to DGM since no angular dependent corrective terms are stored in SGD. However, SGD also introduces inconsistencies when using high order spatial methods for the same reason as DGM. The corrective factor doesn't account for the spatial method used in the fine group problem and causes SGD to converge to a biased fine group solution. These inconsistencies are quite small for the problems tested in literature, but there is no guarantee that they will remain as such for realistic problems. [6][17] 
A second look at SGD, though, reveals that the storage of the second set of cross sections is unnecessary since SGD, as well as recondensation using DGM, can be viewed as an energy multigrid method. Both approaches involve two grids, the fine group energy discretization and the coarse group energy discretization. The fine group problem is collapsed, or restricted, into a coarse group problem which is subsequently solved to provide a coarse group flux. The fine group fluxes from the previous iterate are then updated, or prolonged, using the coarse group flux. One only needs to look at how the SGD cross sections are applied to see how prolongation is implemented.

Once the coarse group eigenvalue problem is solved, the SGD cross sections are multiplied by the new coarse group fluxes to construct the new fine group source. In Equation (2.14), the definition of the SGD cross sections is now expanded within the construction of the new fine group source. The superscript $r$ is now used to differentiate between various parts of the recondensation iteration.

$$
\begin{aligned}
Q_{K}^{r+1}=\frac{1}{2} \sum_{g^{\prime}}( & \left.\frac{\sum_{L \in g^{\prime}} \Sigma_{s, L \rightarrow K} \phi_{L}^{r}}{\phi_{g^{\prime}}^{r}}\right) \phi_{g^{\prime}}^{r+1 / 2} \\
& +\frac{\chi_{K}}{2 k_{e f f}} \sum_{g^{\prime}}\left(\frac{\sum_{L \in g^{\prime}} v \Sigma_{F, L} \phi_{L}^{r}}{\phi_{g^{\prime}}^{r}}\right) \phi_{g^{\prime}}^{r+1 / 2}
\end{aligned}
$$

Definitions for each quantity are provided below:

- $\quad \phi_{\mathrm{L}}^{\mathrm{r}}$ is the scalar flux for fine group $L$ and the current recondensation iteration $r$ produced by the previous decomposition sweep.

- $\phi_{\mathrm{g}}^{\mathrm{r}}$ is the scalar flux for coarse group $g^{\prime}$ calculated by collapsing fine group flux $\phi_{\mathrm{L}}^{\mathrm{r}}$.

- $\phi_{\mathrm{g} \prime}^{\mathrm{r}+1 / 2}$ is the scalar flux for coarse group $g$ ' found by solving the coarse group eigenvalue problem built with coarse group cross sections collapsed using $\phi_{\mathrm{L}}^{\mathrm{r}}$.

- $Q_{K}^{r+1}$ is the source for fine group $K$ to be used for the next decomposition sweep.

When the source is formed according to Equation (2.14), a common factor emerges that scales the fine group flux from the previous recondensation step. This scaling factor is nothing more than the ratio of the new coarse group flux to the initial coarse group flux provided by collapsing the previous iterate of the fine group flux. So instead of defining a separate set of SGD cross sections, a modified fine group flux can be used to produce an equivalent source.

$$
\phi_{K}^{r+1 / 2}=\phi_{K}^{r}\left(\frac{\phi_{g}^{r+1 / 2}}{\phi_{g}^{r}}\right)
$$

$\phi_{K}^{r+1 / 2}$ can then be used in place of the SGD cross sections to construct the fine group source for the next decomposition sweep. The fine group fluxes obtained from the next decomposition sweep represent the fully updated fine group fluxes and would be designated with the $r+1$ index since these are the fluxes used to recalculate the coarse group cross sections. 


\section{Derivation of the Source Equivalence Acceleration Method}

While both DGM and SGD have been limited by certain facets of their approach to solving the fine group problem, they provide key insights into forming a feasible method. Previous work with DGM provided insight into how to correct for spatial inconsistencies when moving to high order spatial methods. However, the exact $\delta$ term in DGM proved to be unwieldy since the original fine group problem is transformed using discrete basis functions. SGD provided a way around this issue since SGD has shown that coarse group fluxes can produce a reconstructed fine group source without the need for discrete basis functions. While SGD did require the production of a second set of scattering cross sections, it was found that this could be simplified by scaling the fine group fluxes from the previous recondensation step using the initial and converged coarse group flux. This approach allows one to use the fine group cross sections directly without the need for additional storage. All of these aspects can be combined to form a feasible method that still provides cheap improvements to the initial solution and does so in a consistent manner when using any spatial method.

To produce a corrective factor that will enforce equivalence between coarse group and fine group equations, the errors introduced from using the coarse group transport coefficients in place of the fine group transport coefficients must be quantified. To take this into account, the fine group transport coefficients in a transport sweep are replaced by the coarse group transport coefficients.

$$
\begin{gathered}
\psi_{\text {out }, l, K}=A_{l, g} \psi_{i n, l, K}+B_{l, g} Q_{K}+\Delta_{l, K}^{i o} \\
\overline{\psi_{l, g, K}}=\frac{\mu_{l}}{d}\left(B_{l, g} \psi_{i n, l, K}+C_{l, g} Q_{K}\right)+\Delta_{l, K}^{a v g}
\end{gathered}
$$

Two corrective terms are added to ensure that no errors are introduced into outgoing and cellaveraged fine group angular fluxes. $\Delta_{l, K}^{i o}$ maintains an equivalent ingoing-outgoing angular flux relationship and $\Delta_{l, K}^{a v g}$ serves the same function for the cell-averaged angular flux relation. However, these terms must be defined according to Equation (3.2) to achieve equivalence.

$$
\begin{gathered}
\Delta_{l, K}^{i o}=\left(A_{l, K}-A_{l, g}\right) \psi_{i n, l, K}+\left(B_{l, K}-B_{l, g}\right) Q_{K} \\
\Delta_{l, K}^{a v g}=\frac{\mu_{l}}{d}\left(\left(B_{l, K}-B_{l, g}\right) \psi_{i n, l, K}+\left(C_{l, K}-C_{l, g}\right) Q_{K}\right)
\end{gathered}
$$

These corrective terms can be thought of as error sources since they deal directly with the difference between the fine group and coarse group transport coefficients. To quantify the errors that would be introduced if corrective terms were not included, all fine group flux quantities (incoming, outgoing and cell-averaged angular fluxes) are expanded in terms of a biased flux, $\tilde{\psi}$, and an error term, $E$, according to Equation (3.3). 


$$
\begin{aligned}
\psi_{\text {in }, l, K} & =\tilde{\psi}_{\text {in }, l, K}+E_{\text {in }, l, K} \\
\psi_{\text {out }, l, K} & =\tilde{\psi}_{\text {out }, l, K}+E_{\text {out }, l, K} \\
\overline{\psi_{l, K}} & =\widetilde{\psi_{l, K}}+E_{\text {avg }, l, K}
\end{aligned}
$$

Since the biased fluxes are produced when no corrective terms are used in Equation (3.1), the biased fluxes can be defined according to Equation (3.4).

$$
\begin{aligned}
& \tilde{\psi}_{\text {out }, l, K}=A_{l, g} \tilde{\psi}_{i n, l, K}+B_{l, g} Q_{K} \\
& \widetilde{\psi_{l, K}}=\frac{\mu_{l}}{d}\left(B_{l, g} \tilde{\psi}_{i n, l, K}+C_{l, g} Q_{K}\right)
\end{aligned}
$$

Equation (3.3) can then be substituted into Equation (3.1) to form Equation (3.5). At this point it is assumed that the source for fine group $K$ does not contain any errors. This is a good assumption since the only reason the source would be erroneous is from using coarse group streaming coefficients in a previous iteration.

$$
\begin{gathered}
\tilde{\psi}_{\text {out }, l, K}+E_{\text {out }, l, K}=A_{l, g}\left(\tilde{\psi}_{i n, l, K}+E_{\text {in }, l, K}\right)+B_{l, g} Q_{K}+\Delta_{l, K}^{i o} \\
\widetilde{\psi_{l, K}}+E_{\text {avg }, l, K}=\frac{\mu_{l}}{d}\left(B_{l, g}\left(\tilde{\psi}_{i n, l, K}+E_{i n, l, K}\right)+C_{l, g} Q_{K}\right)+\Delta_{l, K}^{\text {avg }}
\end{gathered}
$$

However, Equation (3.4) can be used to simplify Equation (3.5) into Equation (3.6).

$$
\begin{gathered}
E_{\text {out }, l, K}=A_{g} E_{i n, l, K}+\Delta_{l, K}^{i o} \\
E_{\text {avg }, l, K}=\frac{\mu_{l}}{d} B_{g} E_{i n, l, K}+\Delta_{l, K}^{\text {avg }}
\end{gathered}
$$

This equation defines an error sweep process using the corrective terms from Equation (3.1) as error sources. If this error sweep is conducted separately from the biased transport sweep, then all that is needed to correct the cell-averaged biased flux is the cell-averaged error term.

$$
\begin{gathered}
\tilde{\psi}_{\text {out }, l, K}=A_{l, g} \tilde{\psi}_{i n, l, K}+B_{l, g} Q_{K} \\
\overline{\psi_{l, K}}=\frac{\mu_{l}}{d}\left(B_{l, g} \tilde{\psi}_{i n, l, K}+C_{l, g} Q_{K}\right)+E_{\text {avg }, l, K}
\end{gathered}
$$

A new $\delta$ term can be formed by collapsing Equation (3.7) into a coarse group problem to produce Equation (3.8). 


$$
\begin{gathered}
\tilde{\psi}_{\text {out }, l, g}=A_{l, g} \tilde{\psi}_{i n, l, g}+B_{l, g} \sum_{K \in g} Q_{K} \\
\overline{\psi_{l, g}}=\frac{\mu_{l}}{d}\left(B_{l, g} \tilde{\psi}_{i n, l, g}+C_{l, g} \sum_{K \in g} Q_{K}\right)+\delta_{l, g}^{a v g} \overline{\phi_{g}}
\end{gathered}
$$

This new $\delta$ term is then defined by Equation (3.9) in which $\overline{\phi_{g}}$ is the coarse group scalar flux obtained by direct collapse of the true fine group scalar flux. However, now that collapse has occurred, the recondensation iteration index $r$ must be included into the definition of the $\delta$ term.

$$
\delta_{l, g}^{a v g, r}=\sum_{K \in g} \omega_{l} E_{a v g, l, K}^{r} / \overline{\phi_{g}^{r}}
$$

Definitions for each quantity are provided below:

- $E_{a v g, l, K}^{r}$ is the error term for angle $l$, fine group $k$ produced by the previous error sweep.

- $\overline{\phi_{g}^{r}}$ is the scalar flux for coarse group $g$ calculated by collapsing fine group flux $\overline{\phi_{K}^{r}}$ produced by the previous fine group sweep.

- $\delta_{l, g}^{a v g, r}$ is the del term for angle $l$ and coarse group $g$ to enforce equivalence with the fine group problem for recondensation iteration $r$.

- $\omega_{1}$ is the quadrature weight for angle $l$.

The cell averaged equation in Equation (3.8) can be integrated over angle through quadrature summation to produce a relationship between the correct coarse group scalar flux, $\overline{\phi_{g}}$, and the biased coarse group scalar flux, $\widetilde{\phi_{g}}$, after a single transport sweep through Equation (3.10).

$$
\overline{\phi_{\mathrm{g}}}=\widetilde{\phi_{\mathrm{g}}} /\left(1-\sum_{\mathrm{l}} \omega_{\mathrm{l}} \delta_{\mathrm{l}, \mathrm{g}}^{\mathrm{avg}, \mathrm{r}}\right)
$$

This shows that a scalar corrective factor is able to drive the biased coarse group scalar flux to the correct coarse group scalar flux. This significantly reduces the memory required to maintain equivalence in the coarse group problem. However, the factor can be further simplified by defining $E_{a v g, l, K}^{r}$ in terms of $\overline{\psi_{l, K}^{r}}$ and $\widetilde{\psi_{l, K}^{r}}$ and then substituting this in for $\delta_{l, g}^{a v g, r}$ in Equation (3.10). The results is Equation (3.11), which defines a scalar multiplicative factor for each cell and coarse group that can correct the biased coarse group flux after each transport sweep at a given recondensation iteration, $r$.

$$
F_{g}^{r}=\frac{\overline{\phi_{g}^{r}}}{\overline{\phi_{g}^{r}}}
$$

One important note is that the biased coarse group scalar flux, $\widetilde{\phi_{g}^{r}}$, used to make $F_{g}^{r}$ must be produced by conducting the coarse group sweep using the collapsed fine group source according 
to Equation (3.12). If any other source is used, then the transport sweeps will not match and $F_{g}^{r}$ term will be incorrectly defined.

$$
\begin{gathered}
\tilde{\psi}_{o u t, l, g}=A_{l, g} \tilde{\psi}_{i n, l, g}+B_{l, g} \sum_{K \in g} Q_{K} \\
\widetilde{\psi_{l, g}}=\frac{\mu_{l}}{d}\left(B_{l, g} \tilde{\psi}_{i n, l, g}+C_{l, g} \sum_{K \in g} Q_{K}\right)
\end{gathered}
$$

Therefore, care must be taken so that the collapsed fine group source is the same one used in the fine group sweep. If any inner iteration (e.g. Gauss-Seidel, upscatter, within-group) is used in the fine group sweep then the converged fine group source from these calculations must be collapsed and used for the coarse group sweep. Otherwise, the fine group source used in the coarse group sweep is not representative of the fine group reaction rates used in the multigroup collapse procedure.

This corrective factor is therefore applied to the flux after a coarse group transport sweep at according to Equation (3.13). In the context of recondensation, this would be applied after a single sweep conducted when solving the coarse group eigenvalue problem at the $r^{\text {th }}$ recondensation iteration.

$$
\phi_{g}^{r+1 / 2, k+1}=F_{g}^{r} \phi_{g}^{r+1 / 2, k+1 / 2}
$$

Definitions for each quantity are provided below:

- $\quad F_{g}^{r}$ is the corrective multiplicative factor for the coarse group $g$ scalar flux.

- $\phi_{g}^{r+1 / 2, k+1 / 2}$ is the scalar flux in coarse group $g$ after coarse group transport sweep $k$.

- $\phi_{g}^{r+1 / 2, k+1}$ is the fully updated scalar flux in coarse group $g$ used to build the source for coarse group transport sweep $k+1$.

Since the fine group source is collapsed for use in the biased coarse group sweep, $F_{g}^{r}$ essentially reproduces the collapsed fine group source in the coarse group problem regardless of how different the coarse group transport coefficients are relative to the fine group transport coefficients. Therefore, this factor is called the Source Equivalence Factor (SEF). Another way to apply SEFs to correct the coarse group sweep is to fold them back into the coarse group source to produce a set of fully consistent coarse group cross sections. This can be done by observing how the SEFs are applied to the biased coarse group scalar flux and then incorporated into the production of the coarse group source. 


$$
\begin{aligned}
Q_{g}^{k+1} & =\frac{1}{2} \sum_{g^{\prime}}\left(\frac{\sum_{K \in g} \sum_{L \in g^{\prime}} \sum_{S, K \leftarrow L} \bar{\phi}_{L}^{r}}{\bar{\phi}_{g^{\prime}}^{r}}\right)\left(\frac{\bar{\phi}_{g^{\prime}}^{r}}{\tilde{\phi}_{g^{\prime}}^{r}}\right) \phi_{g^{\prime}}^{r+1 / 2, k+1 / 2} \\
& +\frac{\chi_{g}}{2 k} \sum_{g^{\prime}}\left(\frac{\sum_{L \in g^{\prime}} v \sum_{f, L} \bar{\phi}_{L}^{r}}{\bar{\phi}_{g^{\prime}}^{r}}\right)\left(\frac{\bar{\phi}_{g^{\prime}}^{r}}{\tilde{\phi}_{g^{\prime}}^{r}}\right) \phi_{g^{\prime}}^{r+1 / 2, k+1 / 2}
\end{aligned}
$$

The most striking observation is that the denominator of the collapsed cross sections cancels with the numerator of these SEFs. Thus, instead of applying the SEFs after each sweep, the coarse group cross sections can instead be defined such that this correction is already taken into account.

$$
\begin{gathered}
v \Sigma_{f, g}(\vec{r})=\sum_{K \epsilon g} v \Sigma_{f, K}(\vec{r}) \bar{\phi}_{K} / \tilde{\phi}_{g}(\vec{r}) \\
\Sigma_{s, g^{\prime} \rightarrow g}(\vec{r})=\sum_{L \in g^{\prime}} \sum_{K \in g} \sum_{s, L \rightarrow K}(\vec{r}) \bar{\phi}_{L}(\vec{r}) / \tilde{\phi}_{g^{\prime}}(\vec{r})
\end{gathered}
$$

Folding the SEFs into these cross sections allows for the simultaneous preservation of cell reaction rates and net leakages without using multiplicative factors after each coarse group transport sweep.

It is important to note that these new cross sections completely replace the original coarse group cross sections; therefore no additional storage is added onto the original coarse group problem. Since the original fine group problem is not modified, the fine group cross sections can still be stored per material as well. There is only a slight increase in memory due to storage of the coarse group scalar flux before and after convergence of the coarse group eigenvalue problem in order to conduct the prolongation of the fine group scalar fluxes. Another slight increase can be attributed to temporary storage of the collapsed fine group source needed to calculate the biased coarse group fluxes. Therefore, these cross sections allow the recondensation process to converge to the fine group solution for any spatial method with very little additional storage. This new approach shares some similarities with SPH methods since it also uses a single multiplicative factor to enforce preservation of reaction rates and net currents [18]. However, the primary difference between SPH factors and SEFs is in the application of these factors to the total cross section. Since SPH factors are applied to the total cross section in addition to scattering and fission, multiple transport sweeps are required to converge these factors. An additional constraint is also required for this approach, typically in the form of a volume-flux normalization equation. For recondensation, use of SPH factors would require an iterative process to converge these factors at each recondensation iteration. [18][19] On the other hand, SEFs are produced assuming that the total cross section isn't modified, therefore all corrections are only being applied to the right-hand side cross sections. Since the total coarse group cross section remains unaffected by the application of these SEFs, only a single fixed source coarse group transport sweep is required at each recondensation iteration to produce them. 


\subsection{Definition of the Source Equivalence Acceleration Method}

The procedure depicted in Figure 1, called the Source Equivalence Acceleration Method (SEAM), incorporates the fully consistent scattering and fission cross sections into the recondensation process. One main difference with this implementation relative to using SGD or DGM is that the collapse of the total cross section can be separated from the collapse of the fission and scattering cross sections. This allows the coarse group fixed source sweep to use the most up to date coarse group total cross section. Use of this cross section takes into account all the errors accrued using the current iterate of the coarse group total cross section as opposed to the previous iterate. 


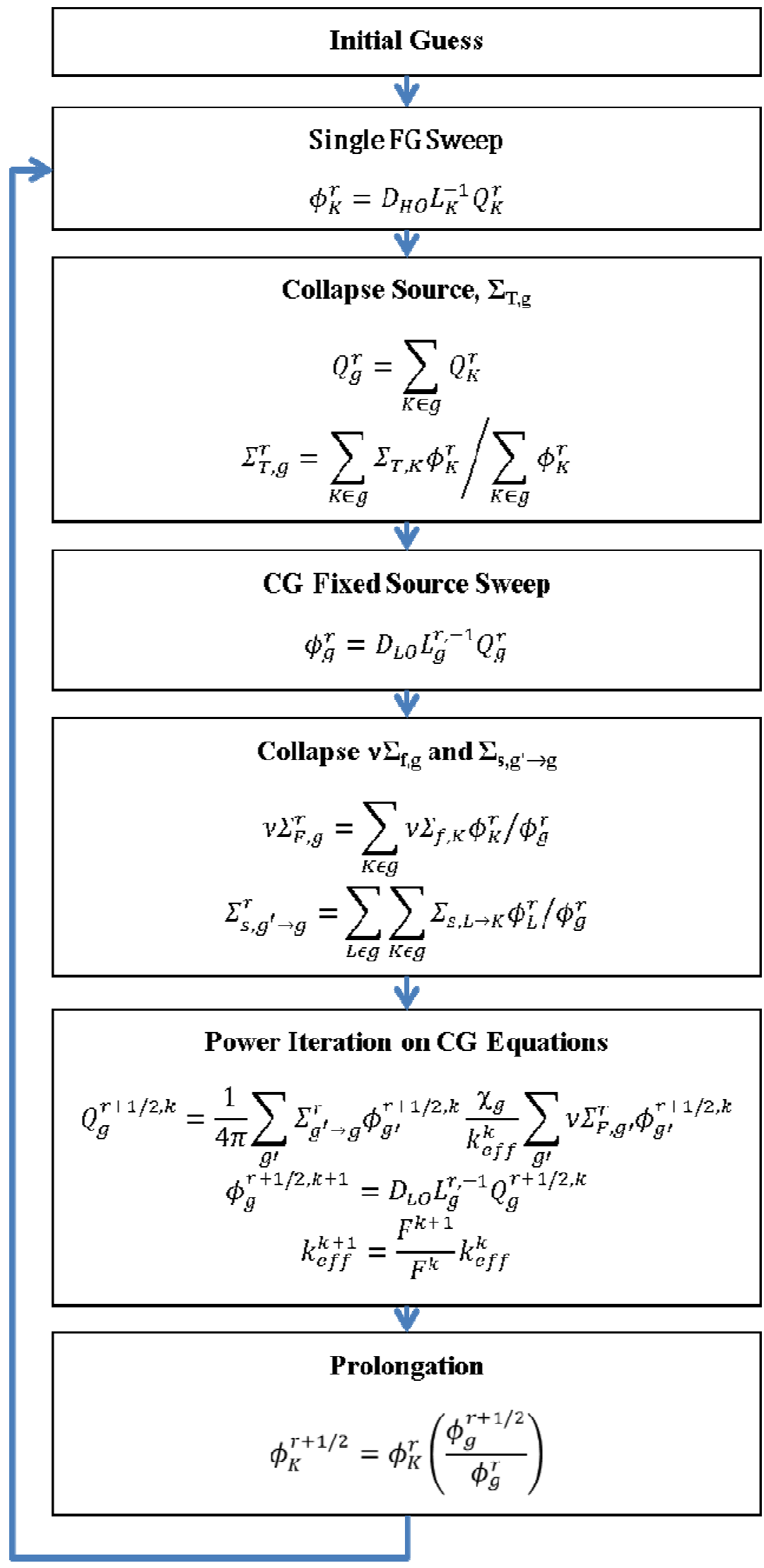

Figure 1 New flow chart for recondensation using the Source Equivalence Acceleration Method (SEAM) 


\section{1D Results}

A 1D reactor model was used to test the 1D application of SEAM and evaluate this method's ability to accelerate fine group transport calculations. The 1D BWR model developed uses 361 group cross sections produced according to the SHEM-361 fine group structure. [15] Though, the cross sections produced are largely unrealistic since no energy self-shielding model was applied in their creation, this core still captures many of the difficult physics involved with the analysis of MOX loaded LWR cores. Key to analyzing this core is being able to capture the swapping of different spectra between neighboring $\mathrm{MOX}$ and $\mathrm{UO}_{2}$ assemblies. While there are slight differences associated with the fission spectra of both assembly types, the true differences come from the presence of different resonances and the larger thermal absorption in the MOX fuel. This 361 group model is able to accurately characterize the spectral shifts potentially lost with a few group model.

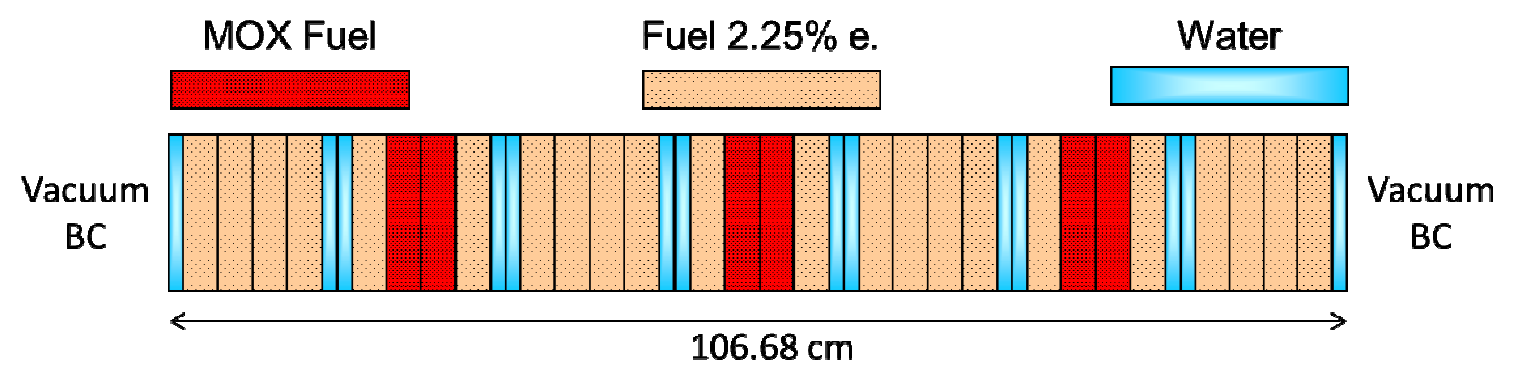

Figure 2 Simple description of the 1D 361 Group BWR Model tested

Figure 2 presents a simple model of the 361 group MOX loaded core used for this analysis. This core consists of an alternating array of 4 low enriched UO2 assemblies and 3 MOX loaded assemblies. These assemblies are $15.24 \mathrm{~cm}$ in width and each contain water spacings separating neighboring assemblies. Vacuum boundary conditions are applied to both sides of the core. Both of these cores were tested in a 1D code built using MATLAB. While MATLAB's built-in functions to do fast matrix-vector products and matrix inversion are advantageous, since it is not a pre-compiled compute language but rather an interpreted language, MATLAB executes instructions directly and translates them on the fly. This leads to very poor performance on transport calculations and is why performance comparison for this section are measured in terms of fine group, coarse group and total number of transport sweeps.

\subsection{Effect of Coarse Group Structure}

In reactor analysis, the choice of coarse group structure is dictated by the ability of said structure to produce a solution accurately relative to the fine group problem. If accuracy is not attained, the coarse group structure is tossed since key physics are likely being neglected. However, in some cases it is not guaranteed that an accurate eigenvalue is truly representative of the accuracy of the whole solution. Depending on the structure analyzed, error cancellations may occur and lead to an erroneously accurate eigenvalue. 
Therefore, recondensation using SEAM is applied to the BWR problem described in Section 4. For each reactor type, two different coarse group structures are chosen for the coarse group problem by applying the methodology proposed by Gibson and Forget. [15] This produced a 34 group structure for the BWR problem. The performances of these structures were compared to a simple 2 group structure. The 2 group structure was chosen such that the second group contained all groups containing upscatter and the first group comprised of the remaining groups. The eigenvalue convergence relative to the reference fine group calculation for each case is compared in Figure 3. For all cases, 1D step characteristics was applied using an $\mathrm{S}_{32}$ angular quadrature.

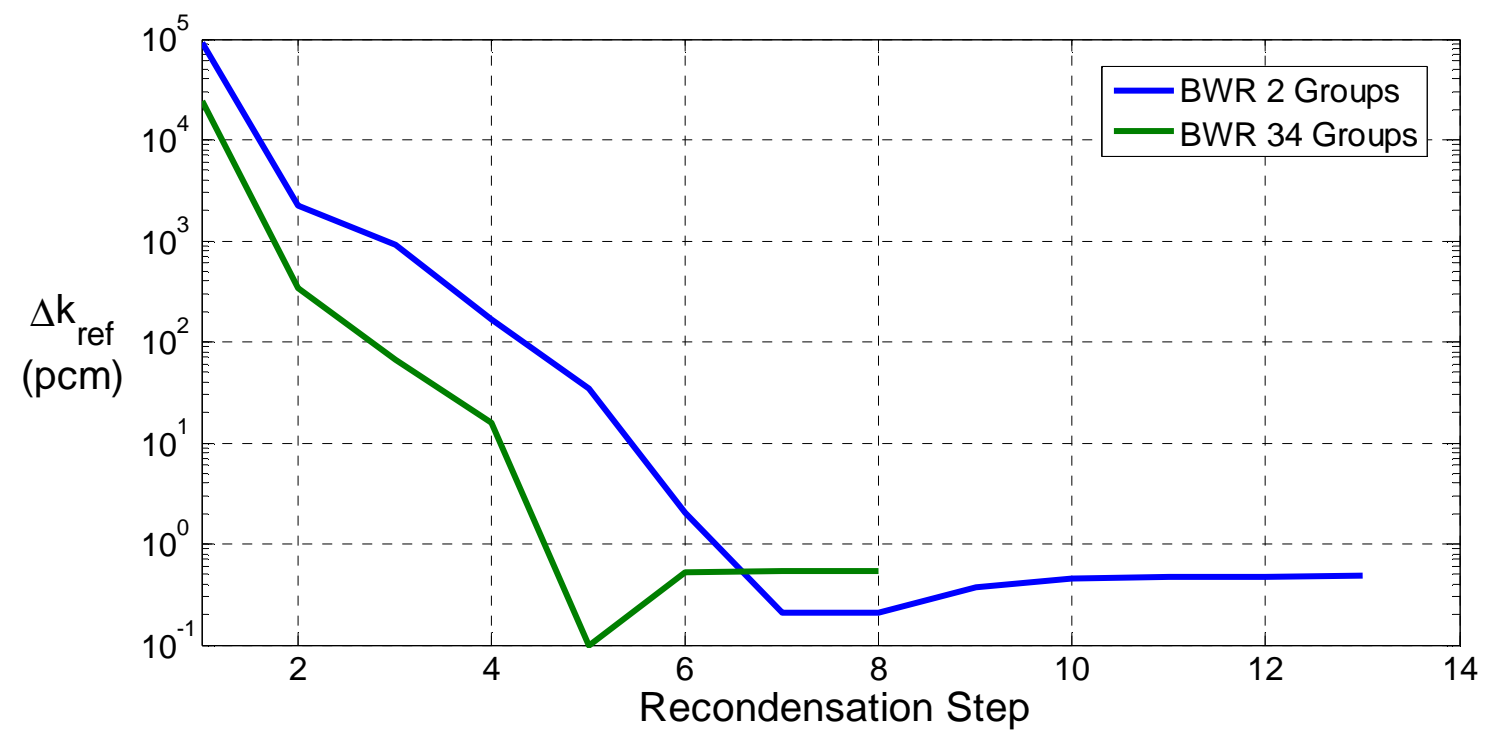

Figure 3 Convergence profiles of SEAM using 2 groups and 34 groups to accelerate the 361 group 1D BWR problem

In the BWR case, the eigenvalues converge to nearly the tolerance applied to the fine group eigenvalue problem. The difference between the recondensation and fine group eigenvalue asymptotically approaches a value below $1 \mathrm{pcm}$ when a convergence criteria of $10^{-7}$ is used. This further shows that the new cross section formulation still maintains full consistency with the fine group problem. When the 34 group structure is used instead of the 2 group, the recondensation process requires fewer recondensation iterations. This is to be expected since the 34 group structure is more likely to capture the physics of the fine group problem while the 2 group structure neglects much of these details. However, since error cancellations can hide the true error of the solution, the error in neutron fluxes for the 34 group and 2 group solutions are calculated and compared relative to the reference fine group solution. 

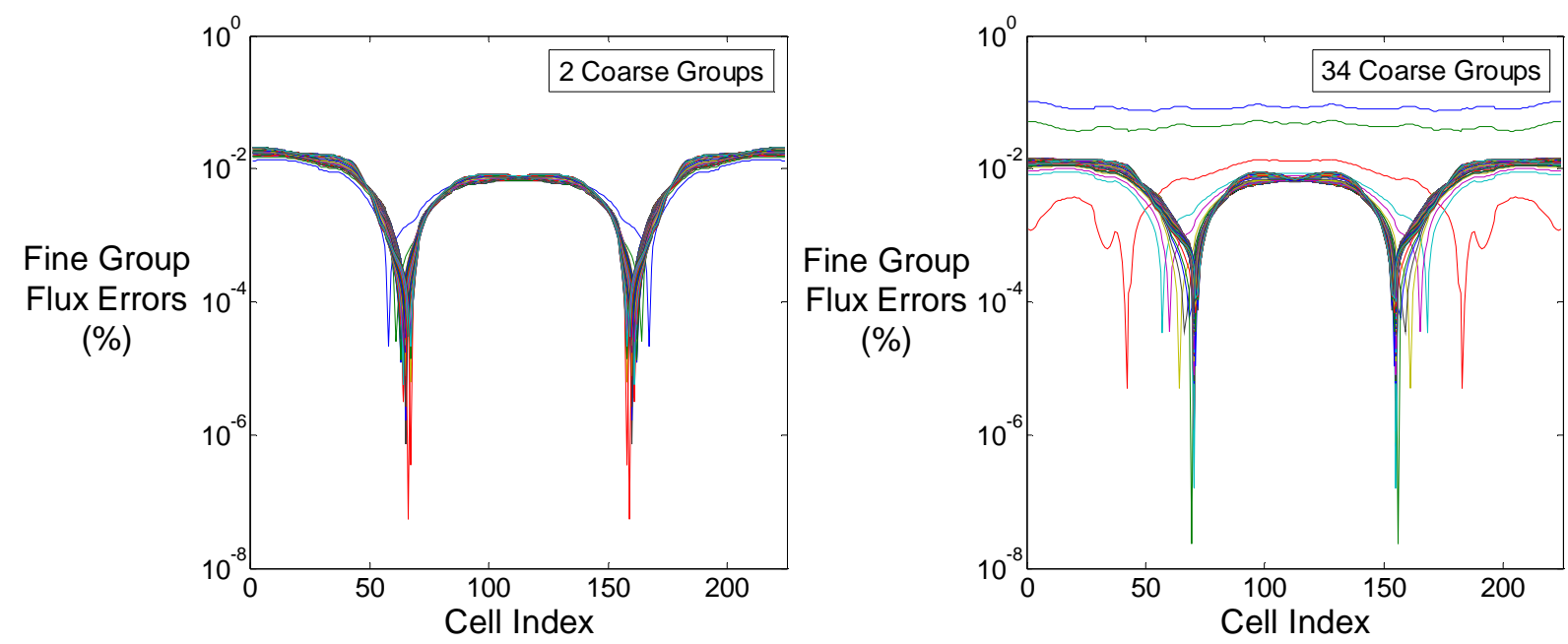

Figure 4 Relative error in the fine group fluxes when using a 2 group and 34 group mapping for the 361 group BWR problem

For both structures, nearly all 361 group fluxes are near or below $0.01 \%$ of the reference solution when using a convergence criteria of $10^{-7}$. Therefore, Figure 4 definitively shows that SEAM is fully consistent and adequately resolves errors such that error cancellation is no longer a concern in our neutronics analyses. The errors in most of the fluxes are so low that the error takes the shape of the second harmonic. This also explains why most of the errors dip so low near the same spatial points because this is where the magnitude of the second harmonic vanishes, leaving a near exact fundamental mode at those points. This has big implications since any coarse group structure may be chosen and consistency with the fine group problem will always be maintained.

It should be noted, though, that errors in 3 of the fine group fluxes for the 34 group structure problem deviate significantly from the other groups. The primary cause is that the magnitudes of these 3 fluxes are on the order of the convergence criteria. Therefore any proportionally large changes between power iterations in these fluxes will not affect satisfaction of the convergence criteria since the maximum true difference in the flux, as opposed to relative difference, defines the residual in this case. Because this quantity required fewer fine group sweeps to satisfy the convergence criteria in the 34 group problem, the errors in these fluxes remained large relative to the other converged fluxes. While the 1D results in this section continue to use this definition of the residual to ensure a fair comparison, the 2D calculations of Section 5 will employ the $L_{2}$ norm of the total source residual to avoid this issue.

With accuracy shown in the BWR case, it is important to determine how much if any computational effort has been spared using this method. For the 2 group and 34 group cases, the total number of coarse group sweeps and fine group sweeps applied are compared in Table 1. These numbers do not take into account the total number of angles being swept over. The number of sweeps applied per fine group is also calculated, as well as the total number of transport sweep which does take into account the total number of angles. 
Table 1 Comparison of transport sweeps for 361 group 1D BWR using different coarse group structures

\begin{tabular}{|c|c|c|c|c|}
\hline CG Structure & CG Sweeps & FG Sweeps & Sweeps/FG & Tot. Sweeps* \\
\cline { 1 - 4 } 2 Group & 2,874 & 10,129 & 28.06 & 208,048 \\
\cline { 1 - 1 } 34 Group & 22,415 & 4,269 & 11.83 & 426,944 \\
\cline { 1 - 1 } Reference & -- & 128,381 & 355.62 & $2,054,096$ \\
\hline
\end{tabular}

* Total sweeps includes number of angles swept - $(\mathrm{CG}+\mathrm{FG}) \mathrm{x}$ Number of Angles

Although the number of coarse group sweeps increases when using the 34 group structure, the number of fine group sweeps is reduced. The result is convergence of the fine group problem, though only 12 full fine group sweeps are applied. For the 2 group problem, 28 sets of fine group sweeps are required to converge. However, the additional effort placed on converging the 34 group problem outweighed the reduction in fine group sweeps in this case, leading to poorer performance relative to the 2 group problem in terms of the total number of transport sweeps. The 2 group problem reduces the number of sweeps by an order of magnitude, while the 34 group problem is only able to reduce this by a factor of 5 .

\section{2D Results using OpenMOC}

While the derivation and testing of SEAM conducted in 1D transport calculations produced very favorable results, this method is of little use if it does not produce the same level of acceleration or better than the methods currently used to accelerate 2D transport calculations. CMFD, for example, has shown great flexibility in its ability to accelerate transport calculations. In OpenMOC, CMFD has been implemented and shown to reduce the number of transport sweeps by nearly a factor of 40 in some cases. [14] The objective of this section, therefore, is to determine SEAM's ability to accelerate fine group 2D transport calculations by implementing it in OpenMOC and comparing its performance to that of CMFD acceleration.

\subsection{A Brief Introduction to OpenMOC}

OpenMOC is an open source 2D Method of Characteristics (MOC) transport solver. [14] Instead of solving for the average angular flux across an entire cell all at once, MOC tallies angular fluxes solved across many tracks superimposed upon the problem. One of the primary simplifications in standard MOC is that the source in each cell is spatially flat. Therefore, each cell in an MOC calculation is a flat source region.

Along each track, the angular flux is calculated using the same process as 1D step characteristics. The incoming angular flux and the source along the direction of the track are used to calculate the outgoing angular flux and the segment average angular flux. The segment is defined as a distinct portion of a single track spanning across a single cell. In order to calculate the full cellaveraged scalar flux, these segment angular fluxes must be integrated across the angular and spatial domain. Exact integration is computationally intractable for complex geometries, so multiple tracks are generated across a set of discrete azimuthal and polar angles to carry out this integration. The Tabuchi-Yamamoto (TY) quadrature is applied to the polar angle in OpenMOC. 
[20] OpenMOC generates these tracks according to a cyclical characteristics condition in order to accurately characterize reflective boundary conditions. Additional information regarding OpenMOC can be found in the associated reference. [14]

\subsection{Step Difference Transport Kernel in OpenMOC}

While many useful features, such as CMFD, have already been added to OpenMOC, some of the methods and approaches desirable for SEAM did not exist in the code. A second transport method, step difference, was added into OpenMOC to test equivalence across differing spatial methods.

While MOC uses the step characteristic definition of the angular flux, in all practicality any method that provides an average angular flux can be used in tallying the scalar flux according to Equation (5.1). Therefore, a step-difference scalar flux tally function was added into OpenMOC to modify the outgoing and average angular flux calculations across a given track segment.

$$
\bar{\psi}=\frac{\mu}{\mu+\tau} \psi_{\text {in }}+\frac{d}{\mu+\tau} Q
$$

In theory, SEAM is able to couple a fine group calculation using MOC to a less expensive transport calculation such as step-difference. This makes the coarse group transport calculation cheaper. Also, since the coarse group total cross sections change after each recondensation step a new exponential interpolation table would likely need to be built. Using SEAM to create an equivalent step difference problem sidesteps this issue by removing the need to calculate exponentials altogether in the coarse group transport sweep.

\subsection{Application of SEAM to the C5G7 Benchmark Core}

The C5G7 benchmark problem is an extremely well documented and analyzed MOX-loaded core commonly analyzed when developing new methods. This benchmark originated primarily as a means to test the ability of codes to analyze non-homogenized problems. [21] In addition to including many of the basic physics of light water reactors, the unstructured nature of this benchmark is ideal for analysis with MOC. In this analysis, the parallel implementation of OpenMOC was used on 12 cores for both CMFD and SEAM. A whole core version of the C5G7 core was used in place of the typical quarter core model. This is the result of a slight inconsistency in how SEAM is applied and how OpenMOC conducts its transport sweep of the core. As it was built for parallelization, the transport sweep is initialized using the boundary angular flux from the previous iteration and is propagated down multiple tracks at once until reach their respective ends. Those same tracks are swept backwards using the boundary angular flux at the opposite end. To enforce consistency using SEAM with this setup, the fine group boundary angular fluxes must also be collapsed at the boundaries in addition to the sources within the flat source regions. Unless the same track spacing and number of azimuthal angles are used, there isn't a clear cut way to ensure that the fine group boundary angular fluxes are collapsed in a consistent manner at, say, a reflective boundary condition. Since a whole core 
uses vacuum boundaries to enforce the same condition across the entire core periphery, this was the ideal approach to implement SEAM in OpenMOC without requiring major changes.

\subsubsection{Energy Acceleration of the C5G7 Benchmark using SEAM}

The C5G7 benchmark problem was first tested using a track spacing of .14 cm and only 4 total azimuthal angles. SEAM was then used to accelerate the normal 7 group problem by creating an equivalent 1 group problem at each recondensation step. The same geometry and tracks were used in the 1 group problem as the 7 group problem. Instead of using MOC with 3 polar angles, the 1 group problem used step-difference with only 1 polar angle.

Since SEAM does not homogenize the spatial problem, there is a one-to-one mapping between the fine group and coarse group geometries. Therefore, a detailed analysis of the fission error distribution can be made to the individual flat source regions.
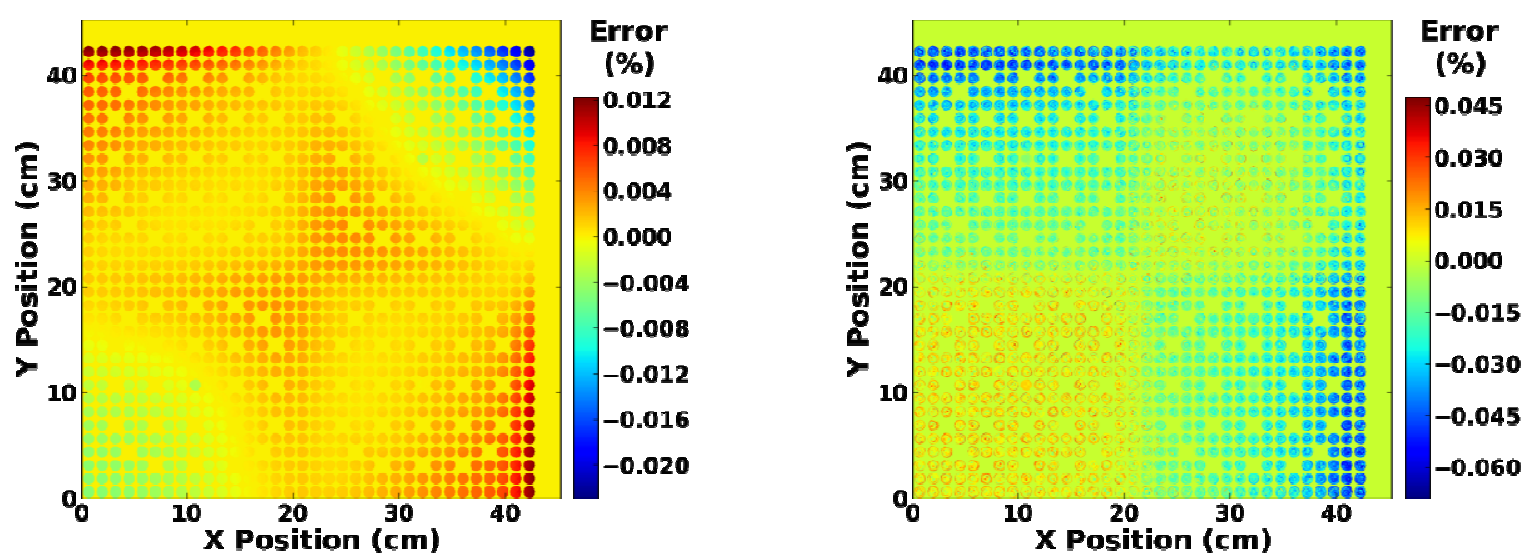

Figure 5 Percentage errors in SEAM 1 Group fission rates using a $10^{-5}$ tolerance relative to the solution using normal power iteration converged with a $10^{-5}$ tolerance (left) and a $10^{-6}$ tolerance (right)

Figure 5 shows that fission rates from SEAM form a near perfect match to those calculated using the normal power iteration on the fine group problem. The errors in the fission source relative to the normal 7 group calculation remain below $0.1 \%$. This is impressive considering the SEAM mostly used power iteration of the 1 group problem with step-difference and only 1 polar angle to converge the 7 group problem.

However, the SEAM calculation did use a source convergence criterion of $10^{-5}$, so it was initially expected that the fission rate errors would drop below .01\%. In order to verify that equivalence has been maintained, the differences in the fission rates of the unaccelerated fine group solution using a source convergence criterion of $10^{-5}$ and $10^{-6}$ were calculated. If the errors from both the SEAM and power iteration calculation using the $10^{-5}$ convergence criteria are similar when calculated relative to the $10^{-6}$ solution, then this provides a good indication that equivalence has been achieved. 


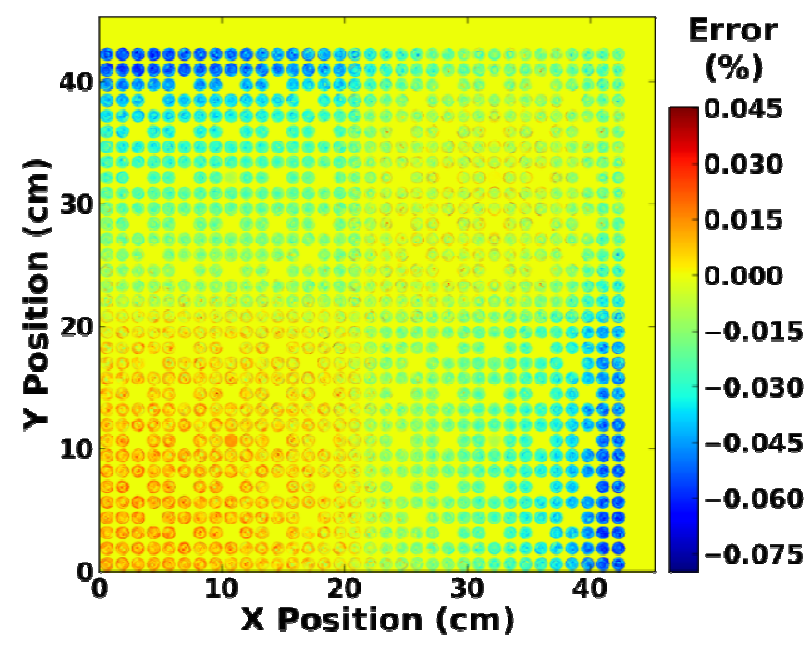

Figure 6 Errors in fission rates using normal power iteration when using a $10^{-5}$ tolerance as opposed to $10^{-6}$ for a 4 azimuthal angle, $0.14 \mathrm{~cm}$ track spacing calculation

The fission rate errors between the two unaccelerated solutions in Figure 6 match very closely to the error distribution on the right side of Figure 5. While the errors are certainly greater than initially expected, the comparison of the unaccelerated and accelerated solutions relative to the more precise solution indicates that equivalence has been maintained in the 1 group calculation using SEAM.

Additional SEAM calculations were conducted using 1,2 and 4 groups to determine the relative impact of coarse group structure on acceleration. This was done by comparing the convergence profiles for all 3 calculations. In each SEAM calculation, the same number of azimuthal angles and track spacing as the 7 group problem were used, but the transport sweep used step-difference and 1 polar angle. A stability issue did arise in subsequent SEAM calculations and for this reason two successive power iterations are used between recondensation steps. This issue will be explained in detail in Section 5.3.2.

The results using SEAM were compared to a 7 Group CMFD accelerated calculation using pincell homogenization. This was deemed to be the best possible acceleration achievable by CMFD for the C5G7 problem since the "coarse" mesh provided a detailed representation of the averaged pin-cell fluxes and the lack of group collapse allowed the spectral effects to be fully accelerated. For this application, the relaxation factor was set to 0.6 to stabilize CMFD acceleration. 


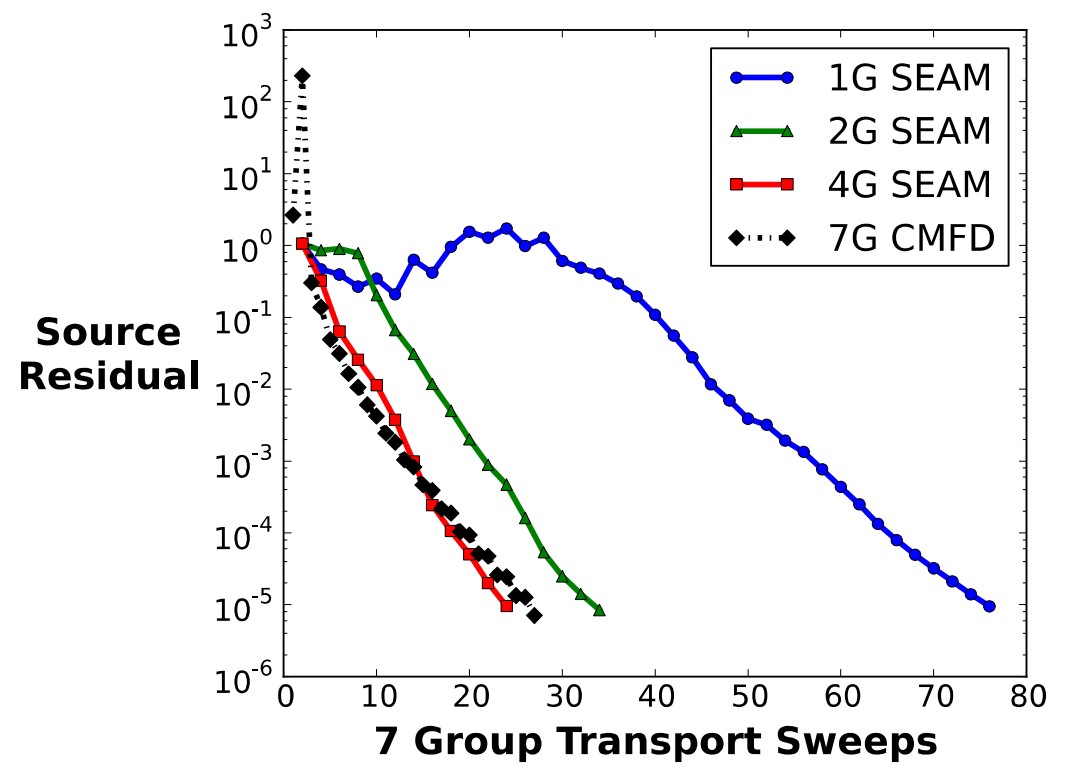

Figure 7 Convergence profiles for SEAM using different coarse group structure and 7 Group CMFD using pin-cell homogenization

As expected, the ability of SEAM to accelerate improves as the number of coarse groups increases. Stability also seems to improve when using more groups. In the 1 group calculation, oscillations occur at first before finally converging and for the 2 group calculation there is an initial stagnation in source residual before convergence begins. When SEAM uses 4 groups, though, convergence is stable and appears to be nearly monotonic. In this case, SEAM is able to converge the solution while only using 24 of the 7 group transport sweeps. This is actually a slight reduction from the 27 sweeps required by CMFD. Although SEAM was able to match the performance of CMFD in the 4 group case, it is important to consider the time required to conduct the power iterations since the transport sweeps used in SEAM are computationally expensive relative to the diffusion calculations of CMFD.

Table 2 Comparison of performance between PI, SEAM and CMFD for the $4 \mathrm{az,} 0.14 \mathrm{~cm}$ C5G7 problem

\begin{tabular}{|c|c|c|c|c|c|}
\hline Method & FG Sweeps & FG Time & CG Sweeps & CG Time & Total Time \\
\hline Normal PI & 768 & 502.6 & $\mathrm{~N} / \mathrm{A}$ & N/A & 502.6 \\
\hline 1 Group & 76 & 51.8 & 6,519 & $1,930.2$ & $2,015.4$ \\
\hline 2 Group & 34 & 21.5 & 1,507 & 563.4 & 601.8 \\
\hline 4 Group & 24 & 15.6 & 939 & 441.1 & 468.4 \\
\hline CMFD & 26 & 19.7 & $26 *$ & 52.4 & 72.1 \\
\hline
\end{tabular}

* The number of diffusion eigenvalue solves used in CMFD

From Table 2 it is seen that the diffusion solve in CMFD is able to beat the SEAM transport sweeps by nearly a factor of 10 . The relatively cheap diffusion calculations in CMFD are key to its success in accelerating transport calculations. Since CMFD is able to create a cheap yet equivalent diffusion problem, SEAM's equivalent transport problem can't compete with regard to total time. 
However, it is important to remember that SEAM is not being used to its full ability in this test case. The reduction in the number of groups is unimpressive and the same number of azimuthal angles and track spacing are being used as in the normal 7 group. It is no surprise that SEAM, at best, barely beats the performance of the normal power iteration. Since SEAM is theoretically able to correct for any inconsistency in the transport sweep operator, it should be possible to incorporate both energy and angular acceleration into the same calculations. If this is the case, then the transport sweeps used in SEAM may be cheap enough relative to a most costly transport problem that the performance could match CMFD.

\subsubsection{Simultaneous Energy-Angle Acceleration}

In previous SEAM calculations, equivalence with the respect to the transport operator was maintained when using different number of polar angles while sweeping across the same tracks. In MOC, though, the accuracy of the transport sweep is also dictated by the number azimuthal angles and the track spacing. Since all of these quantities tie in directly with the transport operator, SEAM should be able to create an equivalent transport problem when using different number of azimuthal angles and track spacings. Therefore, the solution from a 4 azimuthal angle and $0.14 \mathrm{~cm}$ track spacing calculation can be used to accelerate the solution of a 128 azimuthal angle and $0.01 \mathrm{~cm}$ track spacing calculation. As long as the coarser tracks pass through every cell in the problem, then an equivalent transport problem can be formed using SEAM. If this can be achieved, then the transport sweeps used in SEAM become significantly cheaper than the full transport sweep and will improve its performance relative to CMFD.

Initially, this was tested on the C5G7 model with the full transport problem using 2 cases. The first case used 7 groups, 4 azimuthal angles, a $0.14 \mathrm{~cm}$ track spacing, 3 polar angles with MOC. The second case used 32 azimuthal angles, a $0.02 \mathrm{~cm}$ track spacing, 3 polar angles with MOC. With SEAM, an equivalent problem was formed for each using 4 groups, 4 azimuthal angles, $0.14 \mathrm{~cm}$ track spacing, 1 polar angle with step difference. The results using 1 and 2 power iterations per recondensation step were compared for both cases. 


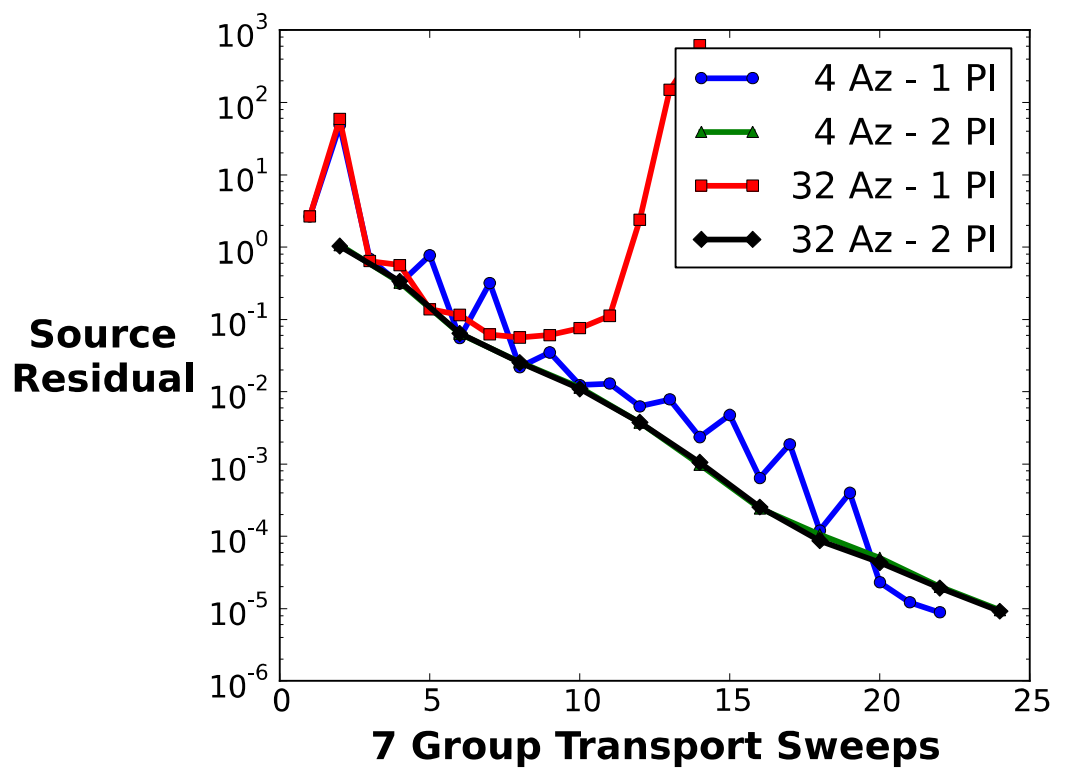

Figure 8 Convergence profiles when using 1 and 2 power iterations between recondensation steps when coupling between similar and dissimilar track layouts in MOC

While using only 1 power iteration was stable for the 4 azimuthal angle case, recondensation diverged for the 32 azimuthal angle problem. At this time, this behavior is not well understood and further investigation is required to understand the mechanism behind this instability. According to Figure 8, using 2 power iterations per recondensation step stabilizes the 32 azimuthal angle problem and dampens out oscillations without significantly increasing the number of full transport sweeps required to converge. This also shows that convergence using SEAM is largely independent of the angular discretization and track spacing of the full transport problem.

However, it still remains to be seen whether the cheaper transport problem SEAM forms is equivalent to the full transport problem when sufficiently converged. Therefore, the flat source region fission rates of the SEAM coarse group solution were compared to the full transport solution using power iteration. This comparison was made with the power iteration solution using a $10^{-5}$ tolerance on the source residual. 


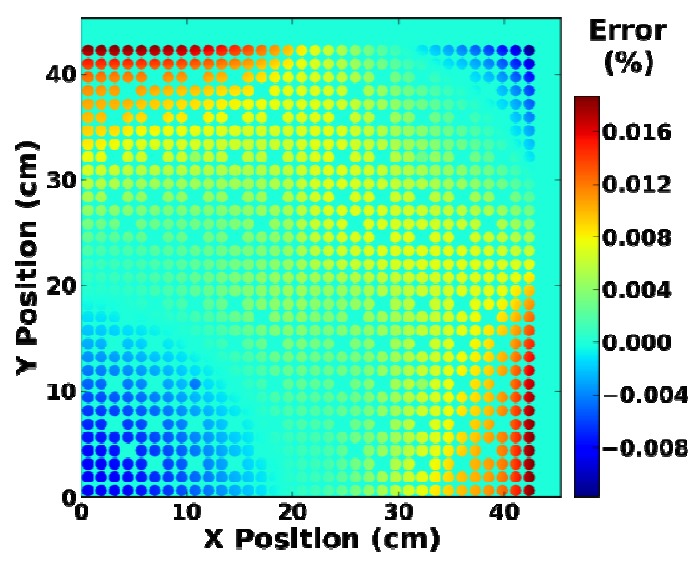

Figure 9 Fission errors of the converged solution using SEAM to accelerate a $32 \mathrm{az}, 0.02 \mathrm{~cm}$ C5G7 model using an equivalent $4 \mathrm{az}, 0.14 \mathrm{~cm}, 4$ group transport problem at each recondensation step. The comparison is made with the full solution converged using $10^{-5}$ as the tolerance for the source residual.

The magnitude of the errors shown in Figure 9 match well with the errors observed in Figure 5. All the local fission rate errors relative to the $10^{-5}$ tolerance calculation remain well below .1\%. These errors shows that SEAM has effectively maintained equivalence using fewer azimuthal angles and wider track spacings than the full transport problem and does so without spatial homogenization.

While macro-track transport-based acceleration of MOC calculations was developed and used in CASMO-4E provide to a similar effect, these macro-tracks are formed using a subset of the finely spaced tracks already being used and therefore require use of the same angular discretization. [22] On the other hand, SEAM builds the low order problem independently of the high order problem, allowing a completely different angular discretization and arbitrary track spacing to be used in the low order transport problem.

The simultaneous group and energy acceleration of SEAM was then tested using different coarse group structures. The same coarse angular discretization and track spacing was used for 1, 2 and 4 group collapse of the $\mathrm{C} 5 \mathrm{G} 7$ core. However, for these tests, the full transport problem used 128 azimuthal angles, a $0.01 \mathrm{~cm}$ track spacing, 3 polar angles, and MOC method. This is the most extreme case tested since the 128 azimuthal angle tracks provide extremely good coverage of a single pin-cell while the 4 azimuthal angle tracks barely covers it. As discussed previously, 2 power iterations are used prior to each recondensation step to ensure stability and near monotonic convergence of SEAM. SEAM's results are compared to that of 7 group CMFD using pin-cell homogenization in Figure 10. 


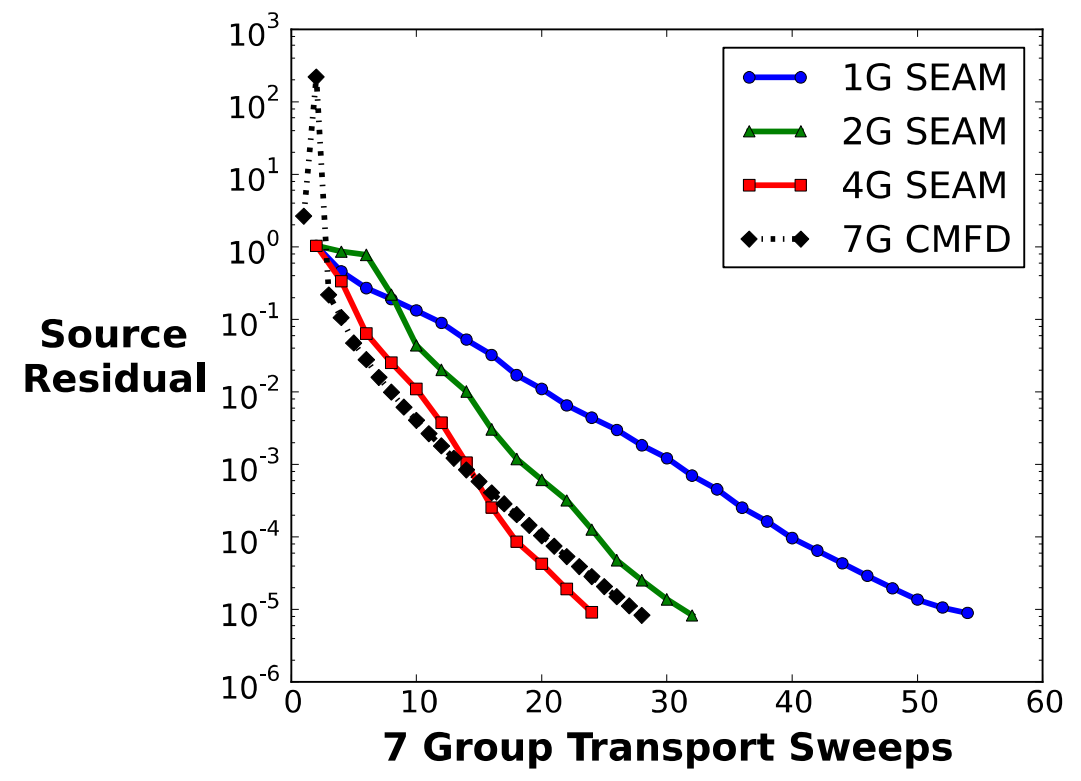

Figure 10 Comparison of convergence profiles for SEAM using 4 azimuthal angles, $0.14 \mathrm{~cm}$ track spacing and for 7 group CMFD using pin-cell homogenization for a 128 azimuthal angle, $0.01 \mathrm{~cm}$ track spacing discretization of the C5G7 problem.

The convergence profiles produced using SEAM on the 128 azimuthal angle C5G7 problem are very similar to the 4 azimuthal angle equivalence. The 1, 2 and 4 group SEAM problems converge with the same number of full transport sweeps or fewer when using 2 power iterations. The performance of each, measured in Table 3, shows that the time to convergence continues to improve for SEAM relative to both the normal power iteration and 7 Group CMFD.

Table 3 Comparison of performance between PI, SEAM and CMFD for the 128 az, $0.01 \mathrm{~cm}$ C5G7 problem

\begin{tabular}{|c|c|c|c|c|c|}
\hline Method & FG Sweeps & FG Time & CG Sweeps & CG Time & Total Time \\
\cline { 1 - 5 } Normal PI & 782 & $45,984.0$ & N/A & N/A & $45,984.0$ \\
\cline { 1 - 1 } 1 Group & 54 & $2,254.2$ & 2,137 & 637.2 & $2,917.8$ \\
\cline { 1 - 2 } 2 Group & 32 & $1,342.2$ & 1,170 & 493.6 & $1,850.6$ \\
\cline { 1 - 2 } 4 Group & 24 & $1,011.4$ & 982 & 479.7 & $1,503.0$ \\
\cline { 1 - 2 } CMFD & 27 & $1,853.7$ & $27 *$ & 55.4 & $1,909.1$ \\
\hline
\end{tabular}

* The number of diffusion eigenvalue solves used in CMFD

The best performance is still achieved when using the 4 group collapse in conjunction with angular acceleration in SEAM. While 7 group CMFD speeds up the calculation by a factor of 24, SEAM is able to achieve a speed up of over 30 relative to the normal power iteration. Although the diffusion eigenvalue problem solved in CMFD still requires nearly 10 times less time than the cheap transport eigenvalue problem used in SEAM, the overhead of the partial current tallies used in CMFD continues to scale proportionally with the number of azimuthal angles and inversely to the track spacing. Therefore, even though the diffusion problem beat SEAM's transport sweeps by 424 seconds, the additional tallies in CMFD accrue roughly an extra 715 seconds. And while CMFD performance could be improved through further 
optimization of the surface tallying function in OpenMOC, it should be noted that SEAM requires no new code to be added into the transport sweep and therefore is much less intrusive to pre-existing codes.

It is apparent from these results that energy acceleration doesn't have a profound impact on the

C5G7 benchmark. Since much of the difficult spectral information has been collapsed down into a few group problem there isn't a significant amount of acceleration which can be achieved through energy acceleration by itself. Therefore, while this work has primarily been focused on energy acceleration, the flexibility and utility of SEAM have been shown to extend far beyond what it was originally intended for. Therefore, it is worth further exploring the possibility of purely angular acceleration for few group problems, meaning the group collapse procedure is not involved in the SEAM calculation.

\subsubsection{Angular Acceleration for Few Group Problems}

The original idea behind the development of SEAM was to be able to create a few group transport method that can equivalently represent the original fine group problem. Ideally, the bulk of the work would be placed on converging the few group problem and reduce the number of fine group transport sweeps. However, in problems already using few groups, there is little to gain from further reducing the number of groups using SEAM. Instead, the results from Section 5.3.2 point towards angular acceleration being the most beneficial for few group problems. Therefore, angular acceleration will be considered when using SEAM with 7 groups. When group collapse is considered, modifying the coarse group cross sections with the corrective factors defined in Section 3 added no additional storage since the coarse group cross sections already needed to be stored for each cell. When using the same number of groups, the corrective factors can instead be applied to the scalar flux after each "incorrect" sweep to produce the equivalent fine group source for the next calculation instead of storing new cross sections for each cell. In this case, only the factors need to be stored for each cell and fine group. Since the storage required for scattering cross sections increases as the square of the number of fine groups, this can significantly reduce storage requirements if only angular acceleration is desirable. However, if one is collapsing the fine group cross sections and storing coarse group cross sections for each cell then using the new cross section definitions in Equation (3.15) may be preferable.

This idea of purely angular acceleration was tested on the C5G7 using SEAM without group collapse but still creating an equivalent transport problem using 4 azimuthal angles, $0.14 \mathrm{~cm}$ track spacing, 1 polar angles and step-difference. This equivalence was formed for the 4, 32 and 128 azimuthal angle C5G7 problems used in Sections 5.3.1 and 5.3.2. Figure 11 shows the results of the SEAM calculations and compares them with the results of 7 group CMFD acceleration using pin-cell homogenization. 


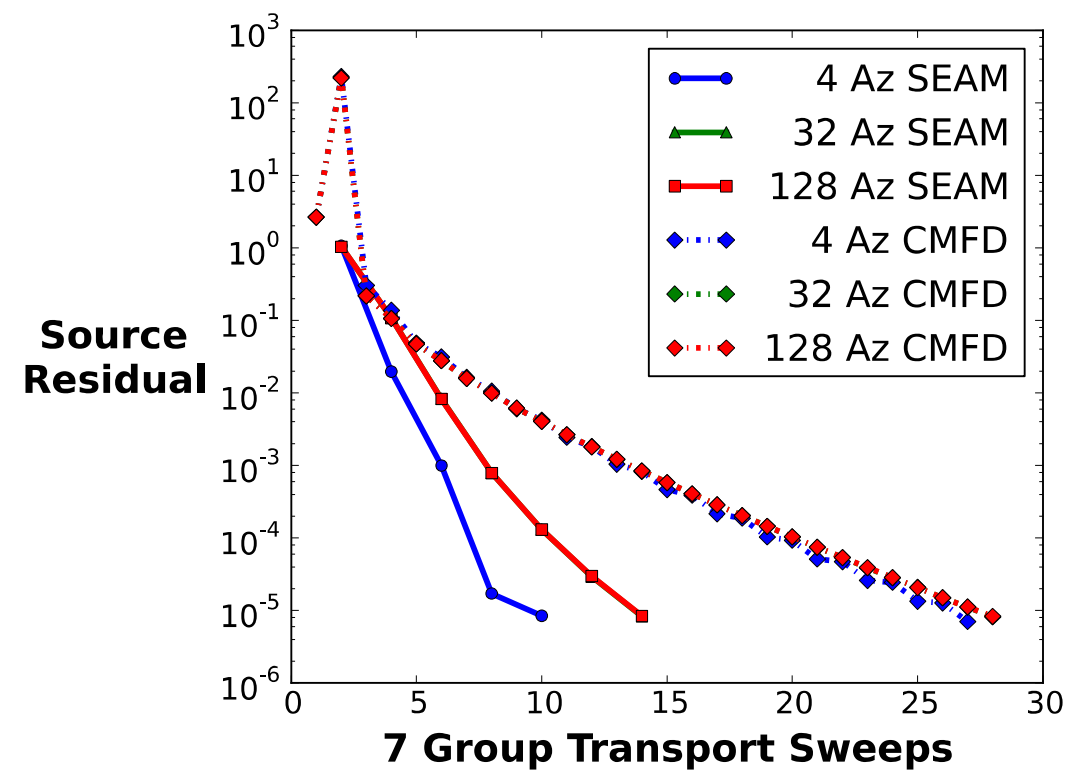

Figure 11 Comparison of convergence profiles for SEAM coupling 4 azimuthal angles, $0.14 \mathrm{~cm}$ track spacing to finer track spacings and for CMFD using pin-cell homogenization, all using 7 groups. All the CMFD runs have the same convergence profile, as does the SEAM calculation for 32 and 128 azimuthal angles.

For C5G7, 7 group SEAM is able to reduce the number of full transport sweeps by almost half relative to 7 group CMFD. The 4 azimuthal angle problem only requires 10 full sweeps, while both the 32 and 128 azimuthal angle problem only require 14. The stellar performance in the 4 azimuthal angle problem is to be expected, however, since fewer approximations are being made. In this case, the same tracks are being used in the full and equivalent transport sweeps, the only difference is that the SEAM transport sweep uses 1 polar angle and step-difference. The true performance measured in time shows that this is still just as time consuming as the normal power iteration. The run time does not significantly improve because the polar angles are included in the SIMD vectorization used in OpenMOC. This means that the sweep process across an individual segment can be carried out simultaneously across all the polar angles at once.

Table 4 Performance of Normal PI, 7 Group SEAM and 7 Group CMFD for the C5G7 Problem

\begin{tabular}{c|ccc|c|c|c}
\hline \multirow{2}{*}{ Method } & \multicolumn{2}{|c|}{ 4 Az. Angles } & \multicolumn{2}{c|}{ 32 Az. Angles } & \multicolumn{2}{c}{ 128 Az. Angles } \\
\cline { 2 - 6 } & Sweeps & Time & Sweeps & Time & Sweeps & Time \\
\cline { 1 - 6 } Normal PI & 768 & 502.6 & 782 & $5,227.4$ & 782 & $45,984.0$ \\
\cline { 1 - 4 } SEAM & $10 / 756^{*}$ & 440.1 & $14 / 845^{*}$ & 670.3 & $14 / 845^{*}$ & $1,110.5$ \\
\cline { 1 - 1 } & 26 & 72.1 & 27 & 330.6 & 27 & $1,909.1$ \\
\hline
\end{tabular}

* (number of full transport sweeps / number of low order sweeps)

The true benefit of angular acceleration with SEAM is seen in the results of the 128 azimuthal angle C5G7 analysis. For this problem, conducting even a few of the full transport sweeps becomes much more expensive than converging the equivalent transport problem. Since 7 group SEAM is able to reduce the number of full transport sweeps by half relative to the CMFD problem, SEAM substantially decreases the time required to converge the solution. For 7 group 
SEAM, the result is a speed up of over 45 relative to the normal power iteration and a speed up of 1.7 relative to CMFD. This could be further improved if the low order solution were more tightly converged at each recondensation iteration.

The primary reason SEAM is able to reduce the number of full transport sweeps relative to CMFD is because SEAM does not make use of spatial homogenization. While CMFD can provide very good acceleration of the global problem, the local problem within each pin-cell can't be effectively accelerated once spatial homogenization takes place. SEAM, on the other hand, performs no spatial homogenization and therefore can accelerate each flat source region individually. In this case, there is no approximation with respect to energy, therefore the group fluxes for each flat source region can be accelerated individually too. This results in a substantial acceleration of the 128 azimuthal angle problem relative to CMFD.

\subsection{Application of SEAM to the "C5G361" Core}

While the C5G7 benchmark served as the ideal problem to initially test SEAM in 2D transport, this problem was not able to provide a good basis for determining the performance of SEAM in a truly fine group problem. Therefore, in order to establish SEAM's ability to accelerate a fine group transport calculation, a modified benchmark problem is proposed that incorporates the 361 group microscopic cross sections used in the 1D BWR analysis of Section 4.1 into the geometry of the original C5G7 benchmark. From here on, this problem will be referred to as the C5G361 problem.

\subsubsection{Description of the "C5G361" Core}

The C5G361 core retains the exact same spatial description of the original C5G7 problem except for a few minor differences. Since the compositions of the guide tubes and fission chambers could not be accurately ascertained from the definition of the C5G7 benchmark description, these materials were replaced with water to simplify the model. Also, due the presence of large resonances with this very detailed fine group data, the number of rings used to describe each fuel pin was increased from 3 to 7 in order to adequately resolve the sharp flux gradients at the resonances. Since the exact compositions of the various $\mathrm{Pu}$ and MA isotopes were also not defined in the C5G7 description, approximate values representative of LWR MOX fuel were used. This 361 group 2D core is the first of its kind to be tested in OpenMOC and should prove difficult enough to test the limits of both SEAM and CMFD in their ability to accelerate fine group transport calculations.

The whole core version of this problem was analyzed using 4 azimuthal angles, $0.14 \mathrm{~cm}$ track spacing and 3 polar angles. As stated before, the fuel rings were divided into 7 concentric, equal area rings and the used the same moderator discretization as in the C5G7 core. However, the guide tubes and fission chambers were replaced with additional moderator using a fine square mesh cell. OpenMOC was used to converge the source residual to a tolerance of $10^{-5}$. The calculated value of $\mathrm{k}_{\text {eff }}$ was 1.180338 . The total run time to converge this problem ended up 
being 86,983 seconds, or just over 24 hours. While the angular discretization used for this problem was very poor, moving to a calculation using 32 angles and a $0.02 \mathrm{~cm}$ spacing would have required roughly 10 days to fully converge. However, the analysis from Section 5.3 indicated that the convergence profiles of both SEAM and CMFD were mostly independent of the angular discretization used in the full transport problem. Therefore, using 4 azimuthal angles should provide a good indication of performance using a more resolved angular discretization.

\subsubsection{C5G361 Fine Group Transport Acceleration with SEAM and CMFD}

For this 361 group 2D problem, two coarse group structures were tested using SEAM. The first is a 9 group structure that largely glosses over many of the prominent resonances except for the $6.67 \mathrm{eV}$ resonance of $\mathrm{U}_{238}$. The 23 group structure separates out the rest of the important resolved resonances to determine if this would significantly improve the convergence of SEAM. The 9 group SEAM calculation was conducted twice, using 1 and 2 power iterations to determine if the behavior observed in Figure 8 was specific to the $\mathrm{C} 5 \mathrm{G} 7$ problem or if it's inherent to SEAM. Each SEAM calculation used the same 4 azimuthal angles and $0.14 \mathrm{~cm}$ track spacing as the original fine group problem, but continued to use step-difference with 1 polar angle for the transport sweep.

These results were then compared to runs using CMFD acceleration. First, pin cell homogenization was used with two different relaxation factors, 0.45 and 0.6 to determine if the cheaper diffusion calculation could provide quick acceleration of the problem. For each relaxation factor, the same coarse group structure as the SEAM calculations were used to conduct 9 group and 23 group calculations with CMFD. The same coarse group structure and the finest possible mesh size were thought to provide a good basis by which to compare SEAM. Most important is to show that both the 9 and 23 group SEAM transport calculations remain equivalent to the full 361 group one. Therefore, the fission rate errors for each flat source region were calculated relative to the converged solution using the normal power iteration procedure in OpenMOC.
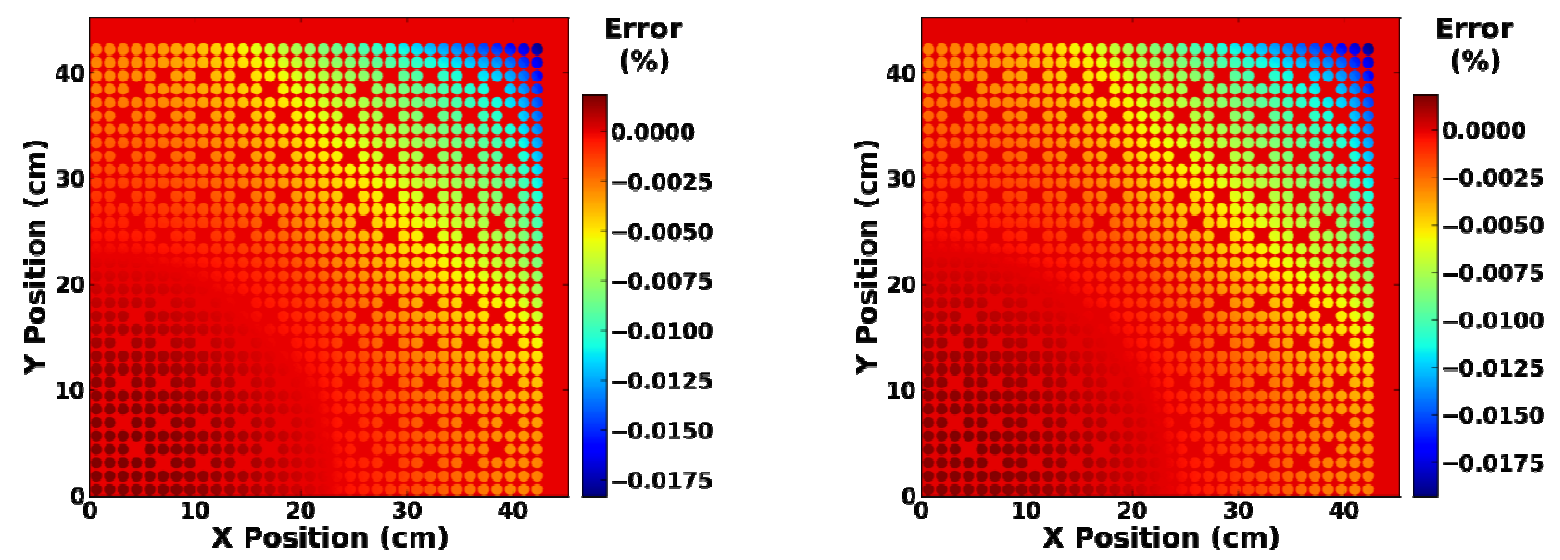

Figure 12 Comparison of fission errors in the 9 group (left) and 23 group (right) problems using SEAM 
Figure 12 shows that all of these errors remain well below $0.1 \%$. In fact, the majority the fission rate errors across over $90 \%$ of the core are below $0.01 \%$ for both the 9 group and 23 group SEAM runs using 2 power iterations per recondensation step. This proves that even for a 361 group problem, SEAM can still create an equivalent transport problem at each recondensation step using significantly fewer groups. With equivalence maintained, the convergence of SEAM will be discussed next.

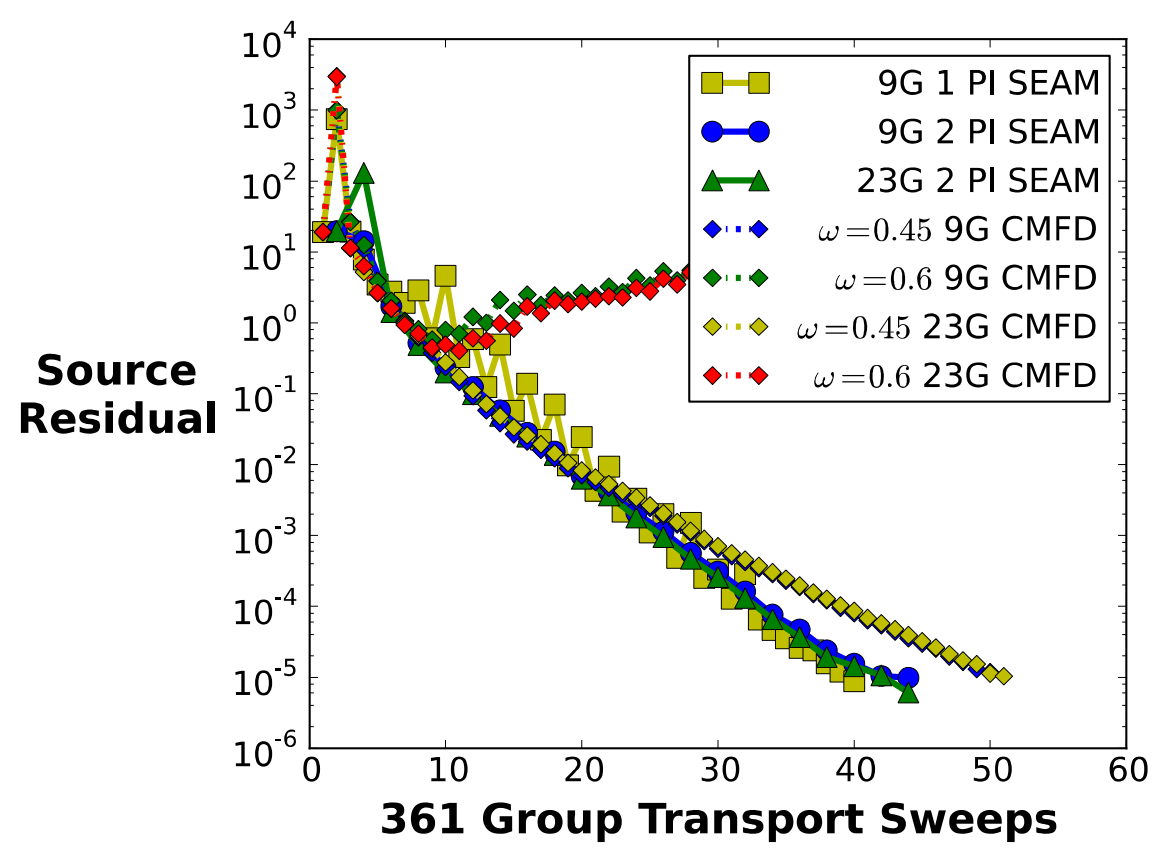

Figure 13 Comparison of convergence profiles for 9 and 23 group SEAM and CMFD (pin cell homogenization) acceleration of the C5G361 problem

Although a very different problem is being considered in this case, oscillations in the convergence of SEAM persist when using 1 power iteration per recondensation step. Since this behavior seems to be problem independent, it is advisable to always conduct 2 power iterations per step to ensure stability and achieve monotonic convergence. Further investigation should be conducted to determine the source of this instability.

Also noticeable in Figure 13 is the near indistinguishable difference in convergence for the 9 group and 23 group SEAM runs using 2 power iterations. Both require 44 fine group transport sweeps before the source is converged. This was somewhat surprising since the 9 group coarse group structure does not provide a detailed representation of the resonances in the 361 group total cross sections. In retrospect, this seems reasonable since any large sources initially present in the resonances at the beginning of the calculation will converge very quickly due to the very quick exponential decay of the flux in resonances. Since this will be picked up very quickly in the 361 group transport sweeps, then it may be important to take other spectral effects, such as upscatter, into consideration when choosing the coarse group structure. While optimal choice of coarse groups is an important step in further optimizing the convergence of SEAM for fine group problems, this subject remains outside the scope of this work. 
Comparison of CMFD and SEAM convergence profiles show that for both the 9 and 23 group calculations is SEAM outpaces CMFD. Both CMFD calculations using a relaxation factor of 0.45 were stable and required 50-51 fine group transport sweeps while SEAM only required 44. While using a larger relaxation factor was thought to further reduce fine group sweeps, the pincell homogenized CMFD calculation became unstable when using the 0.6 relaxation factor.

Further inspection of Figure 13 shows that initially the convergence of CMFD and SEAM match very closely to one another, but when their respective residuals drop below somewhere between $10^{-2}-10^{-3}$ then the convergence of CMFD begins to slow relative to SEAM. Since the coarse group structures, number of azimuthal angles and track spacings are all equivalent, the main difference must lie within the pin cell homogenization used in CMFD. While CMFD is able to produce a much better global solution after each iteration, it eventually has to rely on the fine group transport sweeps to sufficiently converge the spatial dependence of the scalar fluxes within each pin cell. This is likely what's occurring when the convergence of CMFD deviates from SEAM. SEAM, on the other hand, doesn't apply any spatial homogenization and therefore is able to accelerate the scalar fluxes for every flat source region, providing a level of spatial detail to the equivalent problem that CMFD doesn't.

Table 5 Comparison of performance between PI, SEAM and CMFD for the $4 \mathrm{az}, 0.14 \mathrm{~cm}$ C5G361 problem

\begin{tabular}{|c|c|c|c|c|c|}
\hline Method & FG Sweeps & FG Time & CG Sweeps & CG Time & Total Time \\
\hline Normal PI & 2,101 & $86,983.0$ & N/A & N/A & $86,983.0$ \\
\hline 9G SEAM & 44 & $1,752.8$ & 2,290 & $2,383.2$ & $4,457.8$ \\
\hline 23G SEAM & 44 & $1,790.8$ & 2,428 & $4,729.7$ & $6,883.9$ \\
\hline 9G CMFD & 50 & $\begin{array}{c}5,098.6 \\
(3,059)^{* *}\end{array}$ & $50 *$ & $3,673.2$ & $8,771.9$ \\
\hline 23G CMFD & 50 & $\begin{array}{c}4,926.3 \\
(2,963)^{* *}\end{array}$ & $50 *$ & $14,788.7$ & $19,715.0$ \\
\hline
\end{tabular}

* The number of diffusion eigenvalue solves used in CMFD

** Normalization for optimized source construction accounting for overhead

In terms of performance, the 9 group SEAM calculation provides best acceleration for the C5G361 core. SEAM is able to speed up the normal fine group power iteration calculation by a factor of 19.5. On the other hand, CMFD is only able to speed it up by less than a factor of 10 . This is primarily due to how expensive the solving the diffusion problem becomes when doing the full fine group CMFD calculation. The substantial increase in the diffusion solve time moving from 9 groups to 23 groups also highlights the need for optimization for many groups in OpenMOC CMFD solver. Further angular refinement of this problem will also lead to additional overhead in the fine group sweeps for CMFD. All of this taken into consideration, it seems that SEAM is better suited to meet the challenges of fine group transport acceleration. 


\section{Conclusion}

A new method called SEAM was derived through simplifications of the exact $\delta$ term using intuition gained from previous work with DGM conducted at MIT and also from the SGD method developed at Georgia Tech. These simplifications drastically reduced the storage required to produce a fully consistent transport calculation despite the inherent differences in the transport operator when moving from the fine group to coarse group problem. Instead of storing a corrective term for each cell, fine group, and discrete angle, these new factors, SEFs, only need to be stored for each cell and coarse group. These SEFs are also very cheap to produce, only requiring a fixed source coarse group sweep.

The main effect of SEFs was a recreation of the fine group source in the coarse group sweep regardless of how inconsistent the coarse group transport operator is relative to the fine group. Applications of these SEFs to the recondensation process resulted in the nonlinear acceleration method called SEAM. This method was found to be much more general in its application, though, than just correcting for errors accrued in the group collapse process. By definition, the formulation of the SEFs enables the use of any inconsistent method, whether it's a lower order angle approximation or an entirely different transport method altogether from the one used in the fine group calculation.

After testing on a number of 1D fine group cores, SEAM was successfully implemented in OpenMOC and tested on two different 2D problems, the C5G7 benchmark core and a newly created problem called the C5G361 core. Results from C5G7 testing showed that SEAM can create a substantially cheaper transport problem that is still equivalent to the full transport problem. Initial results showed that when moving to large numbers of azimuthal angles like the 128 azimuthal angle example, SEAM was able to outperform CMFD. While SEAM's equivalent transport problem is more expensive to solve, its formation only requires a single fixed source sweep across the low order problem. Therefore, the overhead only scales with the low order problem, not the high order one. On the other hand, the partial current tally overhead in CMFD increases proportionally with the angular order and inversely with track spacing. A point is reached where the increased overhead outpaces the gains from solving the diffusion problem. SEAM was also able to accelerate the $\mathrm{C} 5 \mathrm{G} 7$ problem without using group collapse. The result was a purely angular acceleration approach that further improved the 128 azimuthal angle results. The 7 group SEAM calculation was able to cut the number of full transport sweeps nearly in half relative to the CMFD calculation, resulting in a final speed up of 45 relative to the normal power iteration calculation.

Testing with the C5G7 benchmark core was unable to provide an adequate testing base for a true fine group transport problem, so a new 361 group problem was formed to truly test the performance of energy acceleration using SEAM. This "C5G361" problem was based almost exactly off of the geometry of the C5G7 core, but instead used a 361 group microscopic cross section data set representative of a MOX-load LWR core. This new problem was tested with CMFD using pin cell homogenization to compare with the 9 and 23 group calculation using SEAM. The improvement over CMFD observed in this case suggests that, while the diffusion 
calculation contains a good approximation of the global spatial problem, convergence is limited by changes in the solution at the pin cell level which aren't captured with a homogenized problem. Since SEAM uses no spatial homogenization, the scalar fluxes for each flat source region can be accelerated, leading to the better performance observed for this 361 group problem.

This work only scratches at the surface in terms of applying SEFs and SEAM. One interesting idea is to combine both SEAM and CMFD to take advantage of each's strengths. SEAM's high level of spatial detail gives it the advantage to accelerate each flat source region individually. Unfortunately, this advantage is lost when group collapse is not used since the cost of the acceleration method would be just as high as the fine group transport calculation itself. CMFD, though, can homogenize either at the assembly level or pin cell level to reduce the spatial degrees of freedom and allow it to focus more attention on the spectral problem. Therefore, it should be possible to combine both SEAM and CMFD to achieve better acceleration than either could by themselves. A single whole core transport sweep could be used to set up both the CMFD problem and the SEAM problem. The two could then iterate between each other with CMFD passing improved spectral information to SEAM and SEAM passing improved spatial fluxes to CMFD. This approach has the potential to further reduce the number of fine group whole core transport sweeps required to converge.

Overall, SEAM was shown to accelerate both fine group and high fidelity transport calculations and in many of these cases improve upon the acceleration of CMFD. While further study is merited, it is important to recognize that the recondensation process used in SEAM is nearly identical to that used in DGM. Even though DGM proved ultimately too memory intensive, it still holds valuable intuition into how to further improve SEAM in the future.

\section{Acknowledgements}

This work was supported in part by the Nuclear Engineering University Partnership (NEUP) Fellowship program. This support from the Department of Energy was key to providing the motivation and funding necessary to complete this work.

\section{References}

1. Kim, K. S., \& DeHart, M. D. (2011). Unstructured Partial- and Net-Current Based Coarse Mesh Finite Difference Acceleration Applied to the Extended Step Characteristics Method in NEWT. Annals of Nuclear Energy, 38, 527-534.

2. Park, Y. R., \& Cho, N. Z. (2004). Coarse-mesh Angular Dependent Rebalance Acceleration of the Discrete Ordinates Transport Calculations. Nuclear Science and Engineering, 148, 355-373.

3. Smith, K. S. (1983). Nodal Method Storage Reduction by Non-linear Iteration. Trans. Am. Nucl. Soc., 44(265). 
4. Yamamoto, A. (2005). Generalized Coarse-mesh Rebalance Method for Acceleration of Neutron Transport Calculations. Nuclear Science and Engineering, 151, 274-282.

5. Zhong. Z. et al. (2008). Implementation of Two-level Coarse Mesh Finite Difference Acceleration in an Arbitrary Geometry, Two-dimensional Discrete Ordinates Transport Method. Nuclear Science and Engineering. 158(289).

6. Douglass, S., \& Rahnema, F. (2012). Subgroup Decomposition Method. Annals of Nuclear Energy, 48, 84-101.

7. Rahnema, F., Douglass, S., \& Forget, B. (2008). Generalized Energy Condensation Theory. Nuclear Science and Engineering, 160, 41-58.

8. Zhu, L., \& Forget, B. (2010). A Discrete Generalized Multigroup Energy Expansion Theory. Nuclear Science and Engineering, 166, 239-253.

9. Zhu, L., \& Forget, B. (2011). An Energy Recondensation Method Using the Discrete Generalized Multigroup Energy Expansion Theory. Annals of Nuclear Energy, 38, 17161727.

10. Everson, M. S., \& Forget, B. (2013). Spatial Recondensation using the Discrete Generalized Multigroup Method. Annals of Nuclear Energy, 62, 487-498.

11. Everson, M. S. (2014). Advanced Application of the Discrete Generalized Multigroup Method and Recondensation to Reactor Analysis. Doctor of Philosophy Thesis. Massachusetts Institute of Technology, Cambridge, MA, USA.

12. Lee, D. (2012). Convergence Analysis of Coarse Mesh Finite Difference Method Applied to Two-Group Three-Dimensional Neutron Diffusion Problem. Journal of Nuclear Science and Technology, 49 (9), 926-936.

13. Hong, S. G., Kim, K. S., \& Song, J. S. (2010). Fourier Convergence Analysis of the Rebalance Methods for Discrete Ordinates Transport Equations in Eigenvalue Problems. Nuclear Science and Engineering, 164, 33-52.

14. Boyd, W., Shaner, S., Li, L., Forget, B., \& Smith, K. S. (2014). The OpenMOC Method of Characteristics Neutral Particle Transport Code. Annals of Nuclear Energy, 68, 43-52.

15. Gibson, N. A., \& Forget, B. (2012). Eliminating Flux Updates from the Discrete Generalized Multigroup Method. Proceedings from American Nuclear Society - Winter Meeting. San Diego, CA, USA.

16. Gibson, N. A., \& Forget, B. (2014). On the Stability of the Discrete Generalized Multigroup Method. Annals of Nuclear Energy, 65, 421-432.

17. Yasseri, S., \& Rahnema, F. (2013). Subgroup Decomposition Method in Diffusion Theory. Annals of Nuclear Energy, 60, 235-241.

18. Hebert, A. (1993). A Consistent Technique for the Pin-by-Pin Homogenization of a Pressurized Water Reactor Assembly. Nuclear Science and Engineering, 113, 227-238.

19. Hebert, A. (2009). Applied Reactor Physics. Montreal, QC, CA: Presses Internationales Polytechnique.

20. Yamamoto, A., Tabuchi, M., Sugimura, N., Ushio, T., \& Mori, M. (2007). Derivation of Optimum Polar Angle Quadrature Set for the Method of Characteristics Based on 
Approximation Error for the Bickley Function. Nuclear Science and Technology, 44 (2), 129-136.

21. Nuclear Energy Agency; Organisation for Economic Co-operation and Development. (2003). Benchmark on Deterministic Transport Calculations Without Spatial Homogenization (ISBN 92-64-02139-6). Danvers, MA: Copyright Clearance Center.

22. Smith, K. S., \& Rhodes, J. (2002). Full-Core, 2-D, LWR Core Calculations with CASMO-4E. Proceedings from PHYSOR Conference. Seoul, Korea. 